\title{
LOS ENFOQUES DE BOLTZMANN Y GIBBS \\ FRENTE AL PROBLEMA DE LA IRREVERSIBILIDAD
}

\author{
OLIMPIA LOMBARDI \\ Instituto de Estudios sobre la Ciencia y la Tecnología \\ Universidad Nacional de Quilmes \\ olimpiafilo@arnet.com.ar \\ MaRTín LABARCA \\ Instituto de Estudios sobre la Ciencia y la Tecnología \\ Universidad Nacional de Quilmes \\ mglabarca@unq.edu.ar
}

RESUMEN: El objetivo del presente trabajo consiste en analizar las diferencias entre los enfoques de Boltzmann y de Gibbs respecto del problema de la irreversibilidad. Dicho análisis nos permitirá poner de manifiesto que, en las discusiones acerca de las condiciones necesarias para la irreversibilidad, no suele advertirse que la diferencia central entre los dos enfoques consiste en la utilización de diferentes conceptos de equilibrio y, por tanto, de irreversibilidad. Finalmente se argumentará que, si bien inicialmente ambos enfoques parecen por completo irreconciliables, existen condiciones físicas definidas bajo las cuales los resultados que proporcionan ambos marcos teóricos se aproximan lo suficiente como para ser considerados igualmente admisibles desde el punto de vista de la práctica de la física.

PALABRAS ClAVE: irreversibilidad, Boltzmann, Gibbs, ergodicidad, grados de libertad

SUMMARY: The aim of this paper is to analyze the differences between the approaches of Boltzmann and Gibbs with respect to the problem of irreversibility. This analysis will allow us to show that, in the discussion about the necessary conditions for irreversibility, it goes often unnoticed that the main difference between the two approaches is the use of different concepts of equilibrium and, as a consequence, of irreversibility. Finally, we will argue that, although in principle both approaches seem completely irreconcilable, there are definite physical conditions under which the results provided by both theoretical frameworks are similar enough to be considered equally admissible for all practical purposes.

KEY WORDS: irreversibility, Boltzmann, Gibbs, ergodicity, degrees of freedom

\section{Introducción}

En las exposiciones tradicionales, la mecánica estadística suele ser presentada y explicada como si se tratara de una teoría sistemática, con una formulación única y definitiva que ya no admite disensos: sus problemas se reducirían a meras cuestiones de interpretación respecto de algunos de los conceptos utilizados, como los de probabilidad o ensemble. Sin embargo, este tipo de presentaciones oculta un hecho 
central, responsable de que la mecánica estadística, a más de cien años de su nacimiento, continúe generando profundos debates acerca de sus fundamentos teóricos. En efecto, bajo el rótulo "mecánica estadística" se subsumen dos enfoques, el de Boltzmann y el de Gibbs, que difieren en aspectos teóricos básicos tanto respecto de la explicación del equilibrio, como de la descripción dinámica de la evolución hacia el equilibrio. En particular, ambas posiciones divergen en el modo de dar cuenta del comportamiento irreversible de los sistemas macroscópicos: en el enfoque de Gibbs la ergodicidad es una condición necesaria para la irreversibilidad pero no lo es el alto número de grados de libertad del sistema; mientras que en el enfoque de Boltzmann no es necesario que el sistema sea ergódico para manifestar un comportamiento irreversible, pero el elevado número de grados de libertad sí se considera un requisito indispensable.

En un trabajo anterior (Lombardi 2003) se ha estudiado el papel que cumple la ergodicidad en el marco teórico de Gibbs. El presente trabajo continúa esa línea de investigación: su objetivo consiste en analizar las diferencias entre los enfoques de Boltzmann y de Gibbs sobre la base de los argumentos teórico-formales esgrimidos por los defensores actuales de ambas posiciones antagónicas. Tal análisis permitirá poner de manifiesto los malentendidos conceptuales implícitos en el debate: en la discusión acerca de las condiciones necesarias para la irreversibilidad no suele advertirse que la diferencia central entre los dos enfoques consiste en la utilización de diferentes conceptos de equilibrio y, por tanto, de irreversibilidad en sus respectivas formulaciones teóricas. Finalmente se argumentará que, si bien inicialmente ambos enfoques parecen por completo irreconciliables, existen condiciones físicas definidas bajo las cuales los resultados que ambos esquemas teóricos proporcionan se aproximan lo suficiente como para ser considerados igualmente admisibles desde el punto de vista de la práctica de la física.

\section{Mecánica y termodinámica}

El problema de la irreversibilidad consiste en la pregunta acerca de cómo pueden explicarse las evoluciones macroscópicas irreversibles de la termodinámica en términos de una dinámica microscópica subyacente totalmente reversible. Por lo tanto, el problema se inserta en el marco de los intentos de reducción de la termodinámica a la mecánica, sobre la base del supuesto de que las regularidades macroscópicas resultan de las regularidades que rigen los componentes microscópicos de un sistema. En consecuencia, cuando en este con- 
texto se habla del sistema termodinámico y del sistema mecánico, no se alude a dos entidades independientes, sino a un único sistema bajo diferentes descripciones: como sistema termodinámico $S^{T}$ o como sistema mecánico $S^{M}$.

Puesto que los desacuerdos acerca de las condiciones necesarias para la irreversibilidad descansan sobre argumentos que utilizan el formalismo y los conceptos básicos de la mecánica estadística, el primer paso para analizar este debate con el rigor pertinente consiste en precisar los elementos formales y conceptuales que intervienen en las descripciones mecánica y termodinámica de un sistema físico.

\subsection{Descripción mecánica}

Sea $S^{M}$ un sistema mecánico aislado, esto es, que no intercambia materia ni energía con el medio; por lo tanto, el sistema conserva su energía mecánica $E_{M}$ constante a través del tiempo. Si $S^{M}$ está compuesto por $N$ subsistemas idénticos, cada uno de los cuales posee $n$ grados de libertad - traslacional, rotacional, etc.- , su estado queda determinado por el valor de $2 n N$ variables. Llamando $q_{i}$ a las coordenadas generalizadas y $p_{i}$ a los momentos cinéticos generalizados de cada subsistema, el estado mecánico instantáneo $m(t)$ del sistema $S^{M}$ - microestado mecánico- queda definido por el valor de las $2 n N$ variables de estado:

$$
m(t)=\left(q_{i}(t), p_{i}(t)\right)=\left(q_{1}(t), q_{2}(t), \ldots, q_{n N}(t), p_{1}(t), p_{2}(t), \ldots, p_{n N}(t)\right)
$$

El estado instantáneo de $S^{M}$ suele representarse en el espacio de las fases correspondiente, esto es, una variedad diferenciable de tantas dimensiones como variables de estado posea el sistema. En este caso, se trata de un espacio de las fases $\Gamma$ de $2 n N$ dimensiones, donde cada punto $x(t)$ representa un microestado mecánico posible del sistema.

En el caso particular en el cual $S^{M}$ está compuesto por $N$ partículas puntuales de masa $m$, el estado mecánico de cada partícula queda determinado por el valor de 6 variables: 3 por sus coordenadas posicionales $q_{i}$ y 3 por las componentes de su momento cinético $p_{i}=m d q_{i} / d t$. Por lo tanto, el microestado mecánico instantáneo $m(t)$ de $S^{M}$ queda definido por el valor de las $6 N$ variables de estado:

$$
m(t)=\left(q_{i}(t), p_{i}(t)\right)=\left(q_{1}(t), q_{2}(t), \ldots, q_{3 N}(t), p_{1}(t), p_{2}(t), \ldots, p_{3 N}(t)\right)
$$

En todos los casos, la evolución temporal de $S^{M}$ se encuentra regida por las ecuaciones de Hamilton: 


$$
d q_{i} / d t=\partial H / \partial p_{i} \quad d p_{i} / d t=-\partial H / \partial q_{i}
$$

donde el hamiltoniano $H=H\left(q_{i}, p_{i}\right)$ representa la energía mecánica total $E_{M}$ del sistema $S^{M}$. Las soluciones $q_{i}(t)$ y $p_{i}(t)$ representan la evolución temporal del sistema, dadas las condiciones iniciales $q_{i 0} \mathrm{y}$ $p_{i 0}$. En algunas ocasiones resulta conveniente representar la evolución dinámica del sistema $S^{M}$ mediante un operador $U_{t}$ tal que:

$$
\left(q_{i}(t), p_{i}(t)\right)=U_{t}\left(q_{i 0}, p_{i 0}\right)
$$

En el lenguaje del espacio de las fases, donde $x=\left(q_{i}, p_{i}\right)$ :

$$
x(t)=U_{t} x_{0}
$$

Puede demostrarse que las ecuaciones de Hamilton cumplen las condiciones necesarias para asegurar la existencia y unicidad de sus soluciones para cada condición inicial: ${ }^{1}$ dado $\left(q_{i 0}, p_{i 0}\right)$, existe una única solución $\left(q_{i}(t), p_{i}(t)\right)$ de dichas ecuaciones, solución que describe la evolución mecánica del sistema. En el espacio de las fases $\Gamma$, tal evolución queda representada por una trayectoria $x(t)=\left(q_{i}(t), p_{i}(t)\right)$ que pasa por el punto $x_{0}=\left(q_{i 0}, p_{i 0}\right)$ en $t=0$. Las condiciones de existencia y unicidad también pueden expresarse en lenguaje geométrico: para cada punto representativo del estado inicial, la trayectoria que en él se inicia existe y es única; además, dado que no hay restricciones para fijar el estado inicial del sistema $S^{M}$, las trayectorias no pueden cortarse en ningún punto; es decir, no existe ningún estado mecánico a partir del cual el sistema evolucione temporalmente según dos o más trayectorias posibles.

Dado que $S^{M}$ es un sistema aislado, $H\left(q_{i}, p_{i}\right)=E_{M}$ es una constante de movimiento del sistema, que define una hipersuperficie $\Gamma_{E} \subset \Gamma$ de dimensión $d-1$, donde $d=2 n N$ es la dimensión del espacio de las fases:

$$
\Gamma_{E}=\left\{x=(q, p): H(q, p)=E_{M}\right\}
$$

Por lo tanto, todas las posibles evoluciones del sistema estarán representadas por trayectorias incluidas en $\Gamma_{E}$.

\footnotetext{
${ }^{1}$ Dada una ecuación diferencial ordinaria de primer orden, si se cumplen las siguientes condiciones: (i) puede expresarse en su forma normal $d x / d t=F(x, t)$; (ii) $F(x, t)$ y $d F / d x$ son continuas en un cierto dominio $D$ del plano $O_{x t}$; y (iii) $\left(x_{0}, t_{0}\right)$ es un punto de tal dominio, entonces existe una solución única $x(t)$ de tal ecuación diferencial que satisface $x=x_{0}$ para $t=t_{0}$.
} 
Un importante resultado que se cumple en este tipo de sistemas mecánicos — conservativos - es el teorema de Liouville. Sea $\rho(q, p)$ una función que define una medida $\mu$ sobre $\Gamma$ tal que:

$$
\left\{\begin{array}{l}
\mu(\emptyset)=0 \\
\mu(\Gamma)=1 \\
\text { Si } A \subset \Gamma \text { у } B \subset \Gamma \text { у } A \cap B=\emptyset, \text { entonces } \mu(A \cup B)=\mu(A)+\mu(B)
\end{array}\right.
$$

La medida de un conjunto $A \subset \Gamma$ se define:

$$
\mu(A)=\int_{A} \rho(q, p) d \Gamma \quad \text { donde } d \Gamma=d q_{i} d p_{i}
$$

El teorema de Liouville demuestra que $d \rho / d t=0$, y esto implica que la medida $\mu$ se preserva a través de la evolución:

$$
\mu\left(U_{t} A\right)=\mu(A)
$$

Intuitivamente, si se parte de una función $\rho$ cuyo soporte ${ }^{2}$ se encuentra confinado en una cierta región del espacio de las fases, a través de la evolución tal región inicial puede deformarse y tornarse tan "filamentosa" como para extenderse hasta zonas distantes en el espacio de las fases, pero su volumen permanece siempre constante.

\subsection{Descripción termodinámica}

Sea $S^{T}$ un sistema termodinámico aislado, esto es, que no intercambia materia ni energía con el medio; por lo tanto, su energía termodinámica $U$ se mantiene constante con el tiempo. El estado termodinámico $M(t)$ de $S^{T}$ en el instante $t$ queda definido por un conjunto de variables termodinámicas, entre las cuales pueden distinguirse dos grupos:

- Las variables $C_{k}$ consideradas independientes por cuanto son controlables experimentalmente: su valor se fija externamente con independencia de la situación particular en la que se encuentra $S^{T}$. Tales variables, que funcionan como parámetros, se denominan vínculos [constraints]: ejemplo típico es el volumen de un sistema confinado en un recipiente rígido.

\footnotetext{
${ }^{2}$ El soporte de una función es el subconjunto de su dominio en el cual la función adopta valores no nulos.
} 
- Las variables dependientes $F_{j}$, cuyo valor se ajusta al de las primeras según ciertas relaciones termodinámicas conocidas. Ejemplos de tales variables son la presión y la densidad de un sistema.

Se define como equilibrio termodinámico al estado $M_{e q}$ para el cual las variables termodinámicas $F_{j}$ mantienen su valor constante con el tiempo: $d F_{j(e q)} / d t=0$. Se define la entropía $S$ del sistema en el estado de equilibrio $M_{e q}$ como:

$$
d S=d U / T
$$

donde $T$ es la temperatura absoluta y $d U$ representa un incremento diferencial de energía que conduciría al sistema a través de sucesivos estados de equilibrio.

Empíricamente se observa que cualquier sistema termodinámico aislado $S^{T}$ evoluciona irreversiblemente hacia el equilibrio: las variables termodinámicas $F_{j}(t)$ varían su valor con el transcurso del tiempo hasta alcanzar su valor de equilibrio $F_{j(e q)}$, y a partir de allí mantienen su valor constante; nunca se observa la evolución inversa. En termodinámica se cuenta con distintas ecuaciones diferenciales - por ejemplo, la ley de Fourier de difusión del calor o la ley de Fick de difusión de la materia - cuyas soluciones describen la variación temporal irreversible del valor de las variables termodinámicas $F_{j}(t)$.

El segundo principio de la termodinámica expresa de un modo general la evolución irreversible de los sistemas termodinámicos aislados. Si, al cambiar el valor de los vínculos impuestos, el sistema $S^{T}$ evoluciona desde un estado inicial de equilibrio $M_{e q 1}$ con entropía $S_{1}$ hasta un estado de equilibrio $M_{e q 2}$ con entropía $S_{2}$, se cumple que:

$$
S_{2}>S_{1}
$$

donde $S_{1}$ y $S_{2}$ son funciones de estado del sistema, esto es, no dependen de la particular evolución temporal que describe el sistema al pasar de $M_{e q 1}$ a $M_{e q 2}$.

\subsection{Conexión de ambas descripciones}

Bajo el supuesto de que ambas descripciones refieren a un único sistema, se impone el intento de relacionar de algún modo las variables termodinámicas macroscópicas de $S^{T}$ con las variables mecánicas microscópicas de $S^{M}$, de modo tal que las evoluciones temporales de las $F_{j}$, así como las relaciones entre ellas puedan explicarse en términos de las $q_{i}$ y $p_{i}$ y sus evoluciones temporales. 
El primer paso para conectar ambas descripciones consiste en comprobar que ciertas variables termodinámicas pueden identificarse sin inconvenientes con variables definidas en el plano mecánico. Por ejemplo, el volumen de $S^{T}$, que funciona como vínculo del sistema termodinámico, puede asimilarse al volumen dentro del cual queda confinado el movimiento mecánico de los subsistemas componentes del sistema mecánico $S^{M}$. Sin embargo, en otros casos la relación no es tan directa: es necesario postular una asociación físicamente significativa entre las variables termodinámicas y las variables mecánicas. Un ejemplo sencillo es el de la energía en un sistema aislado: dado que no existe intercambio de energía con el entorno, la energía termodinámica de $S^{T}$ puede asimilarse a la energía mecánica de $S^{M}$ —energía cinética de movimiento más energía potencial de interacción-. Otro ejemplo es el de la presión: si se considera el sistema bajo un modelo mecánico de partículas que colisionan con las paredes del recipiente que las contiene, la presión termodinámica de $S^{T}$ puede asociarse con la velocidad de transferencia de momento cinético a las paredes del recipiente por unidad de área y unidad de tiempo, debida al choque de las partículas de $S^{M}$. En general, se supone que el valor de las variables termodinámicas dependientes $F_{j}$ queda unívocamente determinado por el microestado mecánico del sistema; por lo tanto, a cada $F_{j}$ de $S^{T}$ (con excepción de la temperatura absoluta y la entropía) se asocia una función del estado mecánico de $S^{M}$. En otras palabras, para cada $F_{j}$ se define sobre el espacio de las fases $\Gamma$ una función de fase [phase function] $f_{j}: \Gamma \rightarrow \Re$ tal que asigne a cada punto de $\Gamma$ el valor correspondiente de $F_{j}$. Dada su definición, la función de fase $f(x)$ es una función de muchos-a-uno, pues toma el mismo valor para muchos microestados diferentes, esto es, para muchos puntos del espacio de las fases.

A fin de aclarar los conceptos, consideremos el ejemplo de un gas confinado en la mitad izquierda de un recipiente rectangular de paredes perfectamente aislantes. Empíricamente se observa que, al retirar el tabique divisor entre las dos mitades, el gas comienza a difundirse de modo tal que, luego de un cierto intervalo, acaba distribuyéndose en todo el volumen accesible y en esta situación permanece; nunca se observa la evolución inversa —el gas concentrándose espontáneamente en la mitad izquierda del recipiente-.

- Si se trata de un gas altamente diluido, éste puede ser considerado como un sistema mecánico compuesto por $N$ partículas puntuales de igual masa $m$, cuyas colisiones contra las paredes 
del recipiente son perfectamente elásticas. El microestado mecánico del sistema en el instante $t$ queda, entonces, representado por un punto $x(t)=\left(q_{i}(t), p_{i}(t)\right)$ en el espacio de las fases $\Gamma$ de $6 N$ dimensiones, y su evolución queda representada por una trayectoria en dicho espacio, confinada en la hipersuperficie $\Gamma_{E}$ correspondiente a la energía mecánica total del sistema, $E_{M}$. Al retirarse el tabique divisor entre las dos mitades del recipiente, el movimiento mecánico de las partículas, inicialmente restringido a un volumen $V_{M 1}$, pasa a encontrarse restringido a un volumen $V_{M 2}>V_{M 1}$.

- Según la descripción termodinámica, nos encontramos ante un sistema $S^{T}$, inicialmente en el estado de equilibrio $M_{e q 1}$, confinado en un volumen $V_{T 1}$ y con energía termodinámica $U$; un tiempo después de retirado el tabique divisor, $S^{T}$ adopta un nuevo estado de equilibrio $M_{e q 2}$ con volumen $V_{T 2}$ y manteniendo el valor de $U$ constante. Una variable macroscópica que pone de manifiesto el comportamiento irreversible del sistema es la densidad $\delta$ del gas en la mitad izquierda del recipiente: en el estado de equilibrio inicial, tendrá un valor $\delta\left(M_{e q 1}\right)$; al retirar el tabique divisor, irá disminuyendo con el tiempo hasta alcanzar el valor $\delta\left(M_{e q 2}\right)$ inferior al inicial. Sobre la base del segundo principio puede afirmarse que $S\left(M_{e q 2}\right)>S\left(M_{e q 1}\right)$.

- En este caso, el volumen $V_{T}$ del gas puede identificarse con el volumen $V_{M}$ en el que se encuentra confinado el movimiento mecánico de las partículas $\left(V_{T}=V_{M}=V\right)$. Dado que el sistema se encuentra perfectamente aislado, la energía termodinámica $U$ puede asociarse con la energía mecánica total $E_{M}\left(U=E_{M}=E\right)$. A su vez, la densidad $\delta$ del gas en la mitad izquierda del recipiente en cada instante puede asociarse a la masa total por unidad de volumen en dicha $\operatorname{mitad}, n(t) m /(V / 2)$, donde $n(t)$ es el número de partículas que se encuentran en la mitad izquierda del recipiente en el instante $t$. Dado que $n(t)$ queda unívocamente determinado por el microestado del sistema, puede definirse una función de fase $f_{\delta}(x)$ que adjudica a cada punto del espacio de las fases el valor correspondiente de $\delta$.

Sobre la base de estas herramientas teóricas puede formularse el núcleo del problema de la irreversibilidad: cómo dar cuenta de la evolución termodinámica irreversible de una variable macroscópica $F(t)$ en términos de la evolución mecánica reversible de las microvariables $q_{i}(t)$ y $p_{i}(t)$, esto es, en términos de la trayectoria $x(t)$ que describe 
el punto representativo del microestado del sistema en el espacio de las fases. En el caso de nuestro ejemplo, el problema consiste en explicar la evolución irreversible de la densidad, $\delta\left(M_{e q 1}\right) \rightarrow \delta\left(M_{e q 2}\right)$, en términos de la evolución mecánica irreversible del microestado mecánico $x(t)=\left(q_{i}(t), p_{i}(t)\right)$.

\section{Elementos de teoría ergódica}

El problema que Boltzmann y sus contemporáneos intentaban resolver era el referido a la evolución temporal de los sistemas macroscópicos desde sus estados de no equilibrio hacia el estado de equilibrio termodinámico. Estas cuestiones, que preocupaban a los físicos de fines del siglo XIX, continuaron investigándose a lo largo del siglo XX desde un punto de vista cada vez más formal e independiente de características físicas particulares. Las investigaciones en este campo dieron finalmente origen a lo que hoy se conoce como "teoría ergódica": una teoría matemática que estudia las propiedades estadísticas de los sistemas dinámicos considerados desde un punto de vista totalmente abstracto, esto es, con independencia de la teoría física que describe su evolución temporal (cfr. Lebowitz y Penrose 1973).

En la teoría ergódica, un sistema dinámico queda definido por una terna $\left(\Gamma, \phi_{t}, \mu\right)$ donde:

- $\Gamma$ es el espacio de las fases: espacio topológico considerado generalmente compacto y metrizable.

- $\phi_{t}$ es un flujo dinámico tal que $\phi_{t}: \Gamma \rightarrow \Gamma(t \in \Re)$. $\phi_{t}$ representa la evolución temporal del sistema puesto que convierte el punto $x \in \Gamma$, luego del intervalo $t$, en el punto $x^{\prime}=\phi_{t}(x)$ con $x^{\prime} \in \Gamma$, y análogamente con las regiones de $\Gamma$.

- $\mu$ es una medida normalizada sobre $\Gamma$, invariante bajo el flujo $\phi_{t}$ : para cualquier región $A \subseteq \Gamma$ tal que $\mu(A) \neq 0$, se cumple $\mu\left(\phi_{t}(A)\right)=\mu(A)$. En general, la medida $\mu(A)$ se considera proporcional al volumen de la región $A$; en este caso, la invariancia de $\mu$ bajo $\phi_{t}$ significa que cualquier región de $\Gamma$ evoluciona según $\phi_{t}$ manteniendo su volumen constante a través de la evolución. ${ }^{3}$

\footnotetext{
${ }^{3}$ Como ha sido señalado, en mecánica clásica, esta invariancia de la medida en el espacio de las fases se cumple como consecuencia de la validez del teorema de Liouville.
} 
La teoría ergódica permite clasificar los sistemas dinámicos en las siguientes clases: sistemas ergódicos, sistemas mezcladores, sistemas $K$ y sistemas Bernoulli. Tales clases no son independientes; cada una de ellas incluye a la siguiente. Por lo tanto, existe entre ellas una relación de implicación: si un sistema es mezclador, es ergódico; si es de tipo $K$, es mezclador; si es Bernoulli, es de tipo $K$; pero en ningún caso se da la implicación inversa.

La hipótesis ergódica fue introducida por Boltzmann en 1871; en palabras de su contemporáneo Maxwell, la ergodicidad significa que "el sistema, dejado por sí mismo en su estado de movimiento, tarde o temprano pasará por cualquier fase consistente con la ecuación de energía" (citado en Lebowitz y Penrose 1973, p. 23). En términos actuales, "fase" significa "punto del espacio de las fases"; por lo tanto, en la formulación original, un sistema es ergódico si, dados dos puntos $x$ e $y$ sobre la hipersuperficie de energía constante $\Gamma_{E}$, para algún instante $t$ se cumple $y=\phi_{t}(x)$. En términos aún más sencillos, un sistema es ergódico si el punto representativo de su estado pasa, a lo largo del tiempo, por todos los puntos de $\Gamma_{E}$, sin quedar "atrapado" en ninguna subregión de $\Gamma_{E}$. Por consideraciones dimensionales, hoy se sabe que la hipótesis ergódica en su formulación original no puede ser verdadera: dado que cualquier trayectoria en el espacio de las fases es unidimensional, no puede "cubrir" una hipersuperficie como $\Gamma_{E}$ de dimensión superior a 1. Sin embargo, la idea implícita en las palabras de Maxwell puede conservarse mediante la hipótesis cuasiergódica, que reemplaza "cualquier punto del espacio de las fases" por "cualquier subregión $A$ de volumen finito incluida en $\Gamma_{E}$ " y aclarando que la condición se cumple para casi toda región de $\Gamma_{E}$, excepto para regiones de medida nula. De este modo, se cumple que el punto representativo no permanezca atrapado en ninguna subregión de $\Gamma_{E}$, pero evitando la exigencia demasiado estricta de que pase, a lo largo del tiempo, por todos los puntos de $\Gamma_{E} \cdot{ }^{4}$ Además, en la teoría ergódica actual se ha perdido ya toda referencia a la noción física de energía: $\Gamma_{E}$ es cualquier superficie $S$ del espacio de las fases. Por lo tanto, desde un punto de vista totalmente matemático, la ergodicidad se define del siguiente modo:

\footnotetext{
${ }^{4}$ Esta idea se expresa matemáticamente en términos del concepto de descomponibilidad. Un sistema dinámico es descomponible si el espacio de las fases puede particionarse en dos o más regiones invariantes de medida no nula; por ejemplo, existen $A, B \subset \Gamma_{E}$ tales que $A \cap B=\emptyset, A \cup B=\Gamma_{E}, \mu(A) \neq 0, \mu(B) \neq 0$, y se cumple además que para cualquier $x \in A, \phi_{t}(x) \in A$ y para cualquier $y \in B$, $\phi_{t}(y) \in B$, para todo $t$. Puede demostrarse que un sistema es ergódico sii es no descomponible.
} 
El sistema dinámico $\left(\Gamma, \phi_{t}, \mu\right)$ es ergódico en $S \subset \Gamma$ sii para cualquier región $A \subseteq S$ tal que $\mu(A) \neq 0$, para casi todo $x \in S$ se cumple que $\left\{\phi_{t}(x)\right\} \cap \bar{A} \neq \emptyset$.

Sobre la base del concepto de ergodicidad, en 1931 Birkhoff demostró el llamado teorema ergódico según el cual, para cualquier función $f$ definida sobre el espacio de las fases tal que $f: \Gamma \rightarrow \Re$, el promedio $\langle f(x)\rangle_{p}$ de $f$ sobre la región $S$ del espacio de las fases es igual al promedio temporal $\langle f(x)\rangle_{t}$ de dicha función para $t \rightarrow \infty:^{5}$

$$
\langle f(x)\rangle_{p}=\int_{S} f(x) d \mu=\lim _{T \rightarrow \infty} \int_{0}^{T} f\left(\phi_{t}(x)\right) d t=\langle f(x)\rangle_{t}
$$

La propiedad de mezcla es la siguiente propiedad ergódica, más fuerte que la ergodicidad. Formalmente, la propiedad de mezcla se define del siguiente modo: ${ }^{6}$

El sistema dinámico $\left(\Gamma, \phi_{t}, \mu\right)$ es mezclador en $S \subset \Gamma$ sii para cualesquiera regiones $A, B \subseteq S$, se cumple que $\lim _{|t| \rightarrow \infty} \mu\left(\phi_{t}(A) \cap B\right)=$ $\mu(A) \mu(B)$.

Si bien puede no inferirse de un modo directo de la definición, el hecho de que un sistema sea mezclador en $S$ significa que cualquier región finita $A \subset S$ evoluciona según $\phi_{t}$ manteniendo su medida $\mu(A)$ constante, esto es, su volumen constante, pero deformándose progresivamente; por lo tanto, para $t \rightarrow \infty$, la región inicial puede haberse ramificado sobre toda la región $S$ del espacio de las fases.

En un sistema mezclador, la restricción "para tiempos suficientemente largos", en rigor para $t \rightarrow \infty$, muestra que la mezcla es una propiedad asintótica: no se asegura que la ramificación sea significativa en intervalos finitos de escala macroscópica. ${ }^{7}$ Para que se produzca

${ }^{5}$ Este resultado tiene una importancia central en la mecánica estadística del equilibrio, ya que tradicionalmente se ha supuesto que la igualdad entre el promedio en fase y el promedio temporal explica por qué el promedio en fase brinda una adecuada predicción del valor de la magnitud macroscópica correspondiente en estado de equilibrio. Actualmente, este tradicional supuesto ha sido cuestionado desde diferentes perspectivas ( $c f r$. Friedman 1976, Malament y Zabell 1980, Leeds 1989, Earman y Rédei 1996).

${ }^{6}$ La definición matemática de la propiedad de mezcla fue desarrollada en la década de 1930 por von Neumann y Hopf. Para una presentación formal del tema, véase Arnold y Avez 1968.

${ }^{7}$ Precisamente sobre esta base algunos autores cuestionan el papel del teorema ergódico para calcular el valor de las magnitudes macroscópicas en estado de equilibrio ( $c f r$. Malament y Zabell 1980). Para una discusión acerca del papel de la ergodicidad en la mecánica estadística del equilibrio, cfr. Lombardi 2003. 
una rápida mezcla, las trayectorias que se inician en puntos cercanos del espacio de las fases deben divergir exponencialmente con el tiempo: éste es precisamente el caso de los sistemas $K$. Supóngase que, para un dado sistema, se aplica una partición de grano grueso a su espacio de las fases correspondiente, de modo tal que se divida en celdas de igual volumen; a su vez, se define la macrohistoria del sistema como la secuencia de celdas ocupadas por el punto representativo del estado del sistema a través del tiempo. Si se trata de un sistema $K$, los únicos macroeventos - representados por la ocupación de una celda - que pueden predecirse son los que tienen probabilidad 0 o 1 independientemente de la macrohistoria del sistema.

Los sistemas Bernoulli son un tipo particular de sistemas $K$ en los cuales todos los macroeventos son estadísticamente independientes: la descripción de la macrohistoria completa del sistema no brinda información alguna acerca del próximo macroestado. En otras palabras, las celdas sucesivas se encuentran totalmente no correlacionadas, tal como los números que se obtienen en un juego de azar ideal: la macrohistoria pasada resulta completamente irrelevante respecto de los macroeventos futuros.

\section{El enfoque de Boltzmann}

Es ya un hecho histórico conocido el modo en que Boltzmann fue variando su enfoque teórico a lo largo de su vida como respuesta a críticas a menudo convincentes (cfr. Brush 1976). El propósito aquí no es reconstruir el pensamiento de Boltzmann desde una perspectiva histórica, sino presentar los aspectos centrales del enfoque boltzmanniano tal como son adoptados por sus defensores actuales.

Sea un sistema aislado con energía $E=$ constante. Con independencia del modelo mecánico elegido, puede asegurarse que el sistema sólo puede adquirir microestados representados por puntos pertenecientes a la hipersuperficie $\Gamma_{E}$ de energía $E$. Si además se aplican ciertos vínculos $C_{k}$ al sistema, los puntos correspondientes a los posibles microestados quedan confinados en una superficie $\Gamma_{E C} \subset \Gamma_{E}$ que suele denominarse región accesible del espacio de las fases. En nuestro ejemplo del gas confinado en la mitad izquierda de un recipiente, antes de retirar el tabique divisor sólo serán posibles los microestados para los cuales las posiciones de las partículas se encuentren dentro del espacio físico definido por dicha mitad izquierda.

Sea $F(t)$ una variable macroscópica del sistema y $f(x)$ su función de fase asociada. Cada valor de $F(t)$ define una región $\Gamma(F(t)) \subset \Gamma_{E C}$ tal que: 


$$
\Gamma(F(t))=\left\{x \in \Gamma_{E C}: f(x)=F(t)\right\}
$$

Si $F\left(t_{1}\right) \neq F F\left(t_{2}\right)$, entonces $\Gamma\left(F\left(t_{1}\right)\right) \cap \Gamma\left(F\left(t_{2}\right)\right)=\emptyset$

En otras palabras, $F(t)$ introduce una partición sobre la región accesible del espacio de las fases $\Gamma_{E C}$. Puede considerarse, entonces, que cada elemento $\Gamma(F(t))$ de la partición representa un macroestado $M_{F}(t)$ respecto de la macrovariable $F(t)$.

El núcleo de la propuesta de Boltzmann consiste en asociar la probabilidad de ocurrencia de cada macroestado $M_{F}(t)$ con el número de microestados diferentes compatibles con él; es decir, con el número de puntos pertenecientes a $\Gamma(F(t))$. Pero dado que el conjunto de puntos de cada región $\Gamma(F(t))$ es no numerable, el modo de "contar" microestados consiste en utilizar la medida de Lebesgue $\mu$ definida sobre $\Gamma_{E C}$ que, intuitivamente, mide el volumen de cada subregión de $\Gamma_{E C}$ :

$$
\left\{\begin{array}{l}
\mu(\emptyset)=0 \\
\mu\left(\Gamma_{E C}\right)=1 \\
\operatorname{Si~} \Gamma_{A}, \Gamma_{B} \subset \Gamma_{E C} \text { y } \Gamma_{A} \cap \Gamma_{B}=\emptyset, \text { entonces } \mu\left(\Gamma_{A} \cup \Gamma_{B}\right)=\mu\left(\Gamma_{A}\right)+\mu\left(\Gamma_{B}\right)
\end{array}\right.
$$

Desde esta perspectiva, el macroestado $M_{F}(t)$ más probable será aquel al cual corresponda un mayor número de microestados, es decir, el macroestado representado por la región $\Gamma(F(t))$ de medida máxima, y hacia él tenderá, con alta probabilidad, la macroevolución del sistema. Por lo tanto, desde el enfoque de Boltzmann, el macroestado de equilibrio $M_{F e q}$ se define como el macroestado más probable, esto es, el representado por la región $\Gamma\left(F_{\text {eq }}\right)$ de medida máxima.

De aquí surge la idea de Boltzmann de identificar la entropía de cada macroestado con una medida del número de sus microestados compatibles. En el lenguaje del espacio de las fases, la entropía de Boltzmann se define, entonces:

$$
S_{B}\left(M_{F}(t)\right)=k \log \mu(\Gamma(F(t)))
$$

donde $k$ es la constante de Boltzmann. Dado que la región correspondiente al macroestado de equilibrio es la que posee medida máxima, en cualquier evolución que parte de un macroestado $M_{F}\left(t_{0}\right)$ y se dirige al equilibrio $M_{F e q}$, la entropía, con alta probabilidad, tiende a aumentar, en concordancia con el segundo principio de la termodinámica:

$$
S_{B}\left(M_{F e q}\right)>S_{B}\left(M_{F}\left(t_{0}\right)\right)
$$


Hasta aquí se han conectado las descripciones macroscópica y microscópica para cualquier instante. El paso siguiente consiste en relacionar ambas evoluciones:

- En el micronivel, dado un microestado inicial representado en el espacio de las fases por $x_{0}$ en $t_{0}$, el sistema evolucionará mecánicamente describiendo una trayectoria $x(t)$ en $\Gamma_{E C}$; por lo tanto, la función de fase $f(x)$ describirá una evolución $f(x(t))$ partiendo de su valor inicial $f\left(x_{0}\right)$.

- En el macronivel, dado el valor inicial $F\left(t_{0}\right)$ de la macrovariable correspondiente al macroestado inicial $M_{F}\left(t_{0}\right)$, el sistema evolucionará termodinámicamente según la ecuación de evolución macroscópica para $F(t)$.

¿Qué se requiere para que la macroevolución termodinámica $F(t)$ surja a partir de la microevolución mecánica $x(t)$ ? Se requiere que, no sólo $x_{0}$, sino todos los microestados compatibles con $F\left(t_{0}\right)$ den lugar a microevoluciones que conduzcan al sistema, luego de un tiempo $t$, a microestados compatibles con $F(t)$. En términos de la geometría del espacio de las fases, es necesario que en todos los puntos pertenecientes a $\Gamma\left(F\left(t_{0}\right)\right)$ se inicien trayectorias que, luego de un tiempo $t$, conduzcan al sistema a puntos pertenecientes a $\Gamma(F(t))$. Esta condición se cumple trivialmente en el caso de macrovariables correspondientes a magnitudes que se mantienen constantes con el tiempo, como la energía $E$ del sistema: para todo microestado, $f_{E}\left(x_{0}\right)=f_{E}(x(t))$ y, por tanto, $F_{E}\left(t_{0}\right)=F_{E}(t)$, para todo $t$. El caso interesante es el de las ecuaciones macroscópicas no $t$-invariantes (por ejemplo, Navier-Stokes, Boltzmann, difusión, etc.) que definen macroevoluciones $F\left(t_{0}\right) \rightarrow F_{\text {eq }}$. Aquí debe probarse que, en todos los puntos pertenecientes a $\Gamma_{0}=\Gamma\left(F\left(t_{0}\right)\right)$, se inician trayectorias que, luego de un intervalo suficientemente largo, conducen al sistema a puntos pertenecientes a la región de equilibrio $\Gamma_{e q}=\Gamma\left(F_{e q}\right)$.

Lo dicho hasta aquí puede aplicarse al ejemplo del gas confinado en la mitad izquierda del recipiente, donde la macrovariable $F(t)$ relevante es la densidad $\delta(t)$ en dicha mitad izquierda. En el instante $t_{0}$ en que se retira el tabique divisor, $\delta(t)$ tiene un valor inicial $\delta\left(t_{0}\right)$; la ecuación de difusión describe una macroevolución que tiende al estado de equilibrio $\delta_{\text {eq }}$ luego de un tiempo suficientemente largo: $\delta\left(t_{0}\right) \rightarrow \delta_{e q}$. En este caso debe cumplirse que todas los microestados compatibles con la densidad inicial $\delta\left(t_{0}\right)$ den lugar a microevoluciones que, luego del tiempo considerado, conduzcan a 


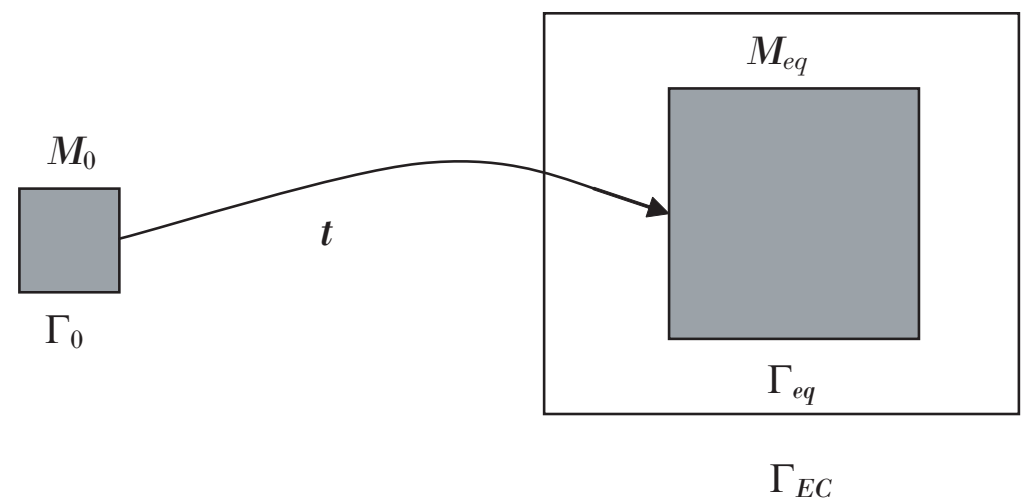

microestados compatibles con $\delta_{e q}$. No siempre es fácil probar que la condición de compatibilidad se cumple, pero puede lograrse en algunos casos particulares; no obstante, ello exige ciertas restricciones fundamentales.

En primer lugar, no es estrictamente cierto que todos los microestados compatibles con $F\left(t_{0}\right)$ den lugar a evoluciones que conducen a microestados compatibles con $F(t)$ : siempre existirán microestados compatibles con $F\left(t_{0}\right)$ que, en lugar de conducir al sistema al macroestado definido por el valor de equilibrio $F_{e q}$, dan origen a evoluciones antitermodinámicas que alejan al sistema del equilibrio. Esto implica cierta restricción respecto de las condiciones iniciales microscópicas del sistema: el enfoque boltzmanniano exige que las condiciones iniciales conflictivas sean suficientemente "atípicas" en el sentido de su ínfima probabilidad de ocurrencia; el volumen que ocupan debe ser inferior en muchísimos órdenes de magnitud al volumen de la región correspondiente al macroestado de equilibrio. Este requisito conduce, a su vez, a otro ingrediente esencial del enfoque de Boltzmann: la necesidad de un elevado número de grados de libertad del sistema.

Si las variables macroscópicas evolucionan hacia su valor de equilibrio, ¿cómo explicar que nunca se observe la evolución inversa? La respuesta se basa en la relación existente entre probabilidad y volumen en el espacio de las fases. El hecho de que las macroevoluciones se dirijan al equilibrio con una altísima probabilidad — que justifique la no observación de evoluciones antitermodinámicas - sólo puede explicarse si la probabilidad del macroestado de equilibrio $M_{F e q}$ es enormemente superior a la probabilidad de cualquier macroestado 
inicial de no equilibrio $M_{F}\left(t_{0}\right)$. Esto supone una enorme disparidad entre los volúmenes de las regiones asociadas: $\mu\left(\Gamma_{e q}\right)>>\mu\left(\Gamma_{0}\right)$. Pero tal desigualdad sólo se cumple en sistemas con un altísimo número de grados de libertad. Éste es el caso de los gases: para un mol de gas en un recipiente de un litro, la relación entre ambas medidas es del orden de $2^{N}$, donde $N$, número de partículas, es del orden de $10^{23}$ —número de Avogadro $\cong 6.10^{23}$ —. Desde el enfoque de Boltzmann, el orden de magnitud de las probabilidades involucradas en este tipo de sistemas permite explicar la irreversibilidad macroscópica observada en los procesos termodinámicos. ${ }^{8}$ A la base de esta posición subyace la idea de la imposibilidad de brindar sentido físico a la distinción micro/macro en sistemas de pocos grados de libertad: así como carece de sentido físico hablar de la temperatura de una única partícula, tampoco parece posible definir variables macroscópicas análogas en la evolución de sistemas de pocos grados de libertad.

Es precisamente el elevado número de grados de libertad de los sistemas de comportamiento irreversible lo que permite a los defensores de la línea boltzmanniana enfrentar la paradoja de Loschmidt. ${ }^{9}$ Llamando $U_{t}$ al operador que expresa la microevolución mecánica del sistema, y $\Gamma_{0}$ a la región correspondiente al macroestado inicial de no equilibrio, luego de un tiempo suficientemente largo, $\Gamma_{0}$ evolucionará mecánicamente hacia una región $\Gamma_{t}$ manteniendo su medida constante de acuerdo con el teorema de Liouville:

$$
\Gamma_{t}=U_{t} \Gamma_{0} \quad \mu\left(\Gamma_{0}\right)=\mu\left(\Gamma_{t}\right)
$$

La paradoja de Loschmidt sostiene que los estados temporalmente invertidos — por inversión de velocidades - de $\Gamma_{t}, \mathbf{T}\left(\Gamma_{t}\right)$, deben evolucionar según $U_{t}$ hacia los estados temporalmente invertidos de $\Gamma_{0}, \mathbf{T}\left(\Gamma_{0}\right)$. Esto efectivamente se cumple puesto que $\mathbf{T}^{-1} U_{t} \mathbf{T} U_{t}=$ $U_{-t} U_{t}=\mathbf{I}$ :

$$
\mathbf{T}\left(\Gamma_{0}\right)=U_{t} \mathbf{T}\left(\Gamma_{t}\right)=U_{t} \mathbf{T}\left(U_{t} \Gamma_{0}\right) \quad \rightarrow \quad \Gamma_{0}=\mathbf{T}^{-1}\left(U_{t} \mathbf{T}\left(U_{t} \Gamma_{0}\right)\right)=U_{-t} U_{t} \Gamma_{0}=\Gamma_{0}
$$

${ }^{8}$ Lavis (2005, pp. 255-256) discute las dificultades que surgen en los intentos de definir el equilibrio desde el punto de vista boltzmanniano en sistemas de pocos grados de libertad.

${ }^{9}$ La paradoja de Loschmidt también suele formularse en los siguientes términos: si en un instante dado de la evolución de un sistema se invierte el sentido de las velocidades de todas las partículas componentes, las colisiones que se producen en sentido inverso "remontan" la evolución original y el sistema vuelve a su estado inicial; de este modo, la entropía disminuirá hasta alcanzar nuevamente su valor inicial. 
Pero el argumento boltzmanniano subraya que los microestados pertenecientes a $\Gamma_{t} \mathrm{y}$, por tanto, también los correspondientes a $\mathbf{T}\left(\Gamma_{t}\right)$, forman un pequeñísimo subconjunto de la región correspondiente al equilibrio $\Gamma_{e q}$ :

$$
\mu\left(\mathbf{T}\left(\Gamma_{t}\right)\right)=\mu\left(\Gamma_{t}\right)=\mu\left(\Gamma_{0}\right)<<\mu\left(\Gamma_{e q}\right)
$$

Por lo tanto, el conjunto de los microestados que resultan de la inversión de velocidades - y que conducirían a una evolución antientrópica- es menos probable, en muchos órdenes de magnitud, que el conjunto de los microestados que conservan el equilibrio. Pero esta enorme disparidad entre las medidas de $\mathbf{T}\left(\Gamma_{t}\right)$ y $\Gamma_{e q}$ sólo se cumple en sistemas de un elevado número de grados de libertad.

El elevado número de grados de libertad de los sistemas de comportamiento irreversible también brinda a los defensores del enfoque de Boltzmann argumentos para resolver la paradoja de la recurrencia: ${ }^{10}$ si se intenta derivar rigurosamente las ecuaciones macroscópicas irreversibles a partir de la dinámica microscópica — más ciertas restricciones acerca de las condiciones iniciales_-, el teorema de recurrencia de Poincaré introduce un fuerte obstáculo. La solución boltzmanniana consiste en diferenciar distintas escalas temporales: una escala en la cual se produce la convergencia hacia el equilibrio, y otra, mucho mayor, en la cual tiene lugar la recurrencia de Poincaré. Pero el tiempo de Poincaré aumenta rápidamente con el aumento del número de partículas del sistema - en particular, tiende a infinito cuando el número de partículas tiende a infinito- - por lo tanto, la diferencia entre ambas escalas temporales se encontrará mejor definida cuanto mayor sea el número de grados de libertad del sistema.

Sobre la base de la presentación de este enfoque, queda claro que la explicación boltzmanniana de la irreversibilidad no requiere que el sistema sea ergódico; sólo exige que todos los microestados compatibles con el valor inicial de la macrovariable considerada conduzcan

\footnotetext{
${ }^{10}$ Mediante las herramientas de la mecánica hamiltoniana, Poincaré demostró que todo sistema mecánico de energía finita, confinado en un volumen finito y compuesto por partículas cuyas interacciones dependen sólo de su posición, luego de un tiempo suficientemente largo retorna indefinidamente cerca del estado inicial; es decir, se comporta cuasiperiódicamente. Tal resultado se conoce como teorema de recurrencia de Poincaré. Sobre la base de este teorema, Zermelo formuló la paradoja de la recurrencia: el segundo principio de la termodinámica no admite demostración mecánica alguna puesto que, mientras tal principio describe la evolución irreversible de un sistema desde un estado de menor entropía hasta el equilibrio de entropía máxima, cualquier sistema mecánico aislado debe volver a una configuración indefinidamente próxima a aquella de la cual partió.
} 
al sistema a microestados compatibles con el valor de equilibrio de dicha macrovariable. Pero esta exigencia es mucho más débil que la condición de ergodicidad, puesto que no establece que el microestado del sistema deba recorrer a través del tiempo toda subregión finita de la región accesible del espacio de las fases.

\section{El enfoque de Gibbs}

La estrategia de Gibbs se basa en describir el comportamiento del ensemble representativo del sistema bajo estudio, esto es, un conjunto de sistemas abstractos, conceptualmente construidos, que poseen la misma microestructura que el modelo mecánico del sistema de interés, están sometidos a los mismos vínculos externos, pero se encuentran distribuidos sobre los diferentes microestados posibles. El microestado instantáneo de cada sistema del ensemble se representa mediante un punto en el espacio de las fases; la situación del ensemble como un todo queda representada por una "nube" de tales puntos representativos, uno por cada sistema del ensemble. Si el número $N$ de sistemas del ensemble es suficientemente alto, la situación del ensemble en cada instante puede especificarse mediante la densidad $\rho(t)=\rho\left(q_{i}, p_{i}, t\right)$ de distribución de los puntos representativos en el espacio de las fases, cumpliéndose que:

$$
N=\int \ldots \int \rho\left(q_{i}, p_{i}, t\right) d q_{i} d p_{i}
$$

En general, $\rho$ se considera normalizada a la unidad:

$$
1=\int \ldots \int \rho\left(q_{i}, p_{i}, t\right) d q_{i} d p_{i}
$$

En este caso, $\rho$ es una medida que brinda la probabilidad por unidad de volumen de que un punto representativo de un sistema cualquiera del ensemble se encuentre en las diferentes regiones del espacio de las fases.

La función $\rho$ hace posible calcular los promedios, sobre todos los sistemas del ensemble, de cualquier magnitud que dependa de los microestados de tales sistemas. Por ejemplo, si consideramos una magnitud mecánica que define una función de fase $f: \Gamma \rightarrow \Re$, su promedio en fase $\langle f(x)\rangle_{p}$ en un instante dado se calcula:

$$
\langle f(x)\rangle_{p}=\left\langle f\left(q_{i}, p_{i}\right)\right\rangle_{p}=\int \ldots \int f\left(q_{i}, p_{i}\right) \rho\left(q_{i}, p_{i}\right) d q_{i} d p_{i}=\int f(x) \rho(x) d x
$$

Desde este enfoque, se define equilibrio estadístico como la situación en la cual la probabilidad representada por $\rho$ y los promedios 
en fase son independientes del tiempo. Un modo sencillo de asegurar el equilibrio estadístico es construir un ensemble para el cual $\rho$ se distribuye uniformemente sobre todo el espacio de las fases:

$$
\rho\left(q_{i}, p_{i}\right)=c t e \quad \rightarrow \quad \partial \rho / \partial q_{i}=\partial \rho / \partial p_{i}=0 \quad \rightarrow \quad d \rho / d t=0
$$

En particular, para representar un sistema aislado — donde $E$ es una constante de movimiento - Gibbs recurre al ensemble microcanónico, que suele definirse de un modo totalmente general como:

$$
\begin{cases}\rho(x)=c t e & \text { para } x \in \Gamma_{E} \\ \rho(x)=0 & \text { para } x \notin \Gamma_{E}\end{cases}
$$

Sin embargo, cuando nos referimos a un sistema aislado en particular, con los vínculos $C_{k}$ impuestos externamente, sus microestados posibles no son los representados por los puntos pertenecientes a $\Gamma_{E}$ : los sistemas del ensemble deben distribuirse uniformemente sobre los posibles microestados representados por puntos pertenecientes a $\Gamma_{E C} \subset \Gamma_{E}$, región accesible del espacio de las fases. Por lo tanto, en cada situación, dados los particulares vínculos impuestos al sistema bajo estudio, el ensemble microcanónico se define como:

$$
\begin{cases}\rho(x)=c t e & \text { para } x \in \Gamma_{E C} \\ \rho(x)=0 & \text { para } x \notin \Gamma_{E C}\end{cases}
$$

En este caso, el promedio en fase de cualquier función de fase $f(x)$ se calcula como:

$$
\langle f(x)\rangle_{p}=\int_{\Gamma E C} f(x) \rho(x) d \Gamma
$$

En otras palabras, a cada variable macroscópica $F(t)$ del sistema termodinámico $S^{T}$ —con excepción de la entropía y la temperatura absoluta - corresponderá una función de fase $f(x)$ sobre el espacio de las fases de $S^{M}$ tal que, para el ensemble microcanónico representado por $\rho(x)$, se cumple:

$$
F_{e q}=\langle f(x)\rangle_{p}=\int_{\Gamma E C} f(x) \rho(x) d \Gamma
$$

Por su parte, en estado de equilibrio estadístico, la entropía de Gibbs $S_{G}$ se calcula como:

$$
S_{G}=-k \int_{\Gamma} \rho(x) \log \rho(x) d \Gamma
$$


¿Cómo dar cuenta, desde la perspectiva gibbsiana, de la macroevolución de un sistema hacia el equilibrio termodinámico? Supóngase un sistema aislado, sometido a ciertos vínculos $C_{0}$, inicialmente en equilibrio termodinámico; en esta situación, el sistema está representado adecuadamente por un ensemble microcanónico definido por:

$$
\begin{cases}\rho(x)=\text { cte } & \text { para } x \in \Gamma_{E C 0} \\ \rho(x)=0 & \text { para } x \notin \Gamma_{E C 0}\end{cases}
$$

y la entropía de Gibbs será $S_{G}$. Si en $t_{0}$ se modifican los vínculos aplicados al sistema - por ejemplo, en el caso del gas confinado en la mitad izquierda del recipiente, se elimina el tabique divisor-, el macroestado originalmente de equilibrio se convierte en un macroestado de no equilibrio que evoluciona hacia un nuevo macroestado de equilibrio determinado por los nuevos vínculos $C_{1}$ impuestos al sistema. En la descripción de Gibbs, el ensemble microcanónico original representado por $\rho(x)$ se convierte, en $t_{0}$, en un ensemble de no equilibrio representado por $\rho_{0}(x, t)$, cuyo soporte se encuentra confinado a $\Gamma_{E C 0}$. Este ensemble de no equilibrio debería evolucionar hacia un nuevo ensemble microcanónico definido por:

$$
\begin{cases}\rho_{1}(x)=c t e & \text { para } x \in \Gamma_{E C 1} \\ \rho_{1}(x)=0 & \text { para } x \notin \Gamma_{E C 1}\end{cases}
$$

donde $\Gamma_{E C 1}$ es la nueva hipersuperficie accesible dados los nuevos vínculos impuestos, y siendo $\Gamma_{E C 1}>\Gamma_{E C 0}$. En otras palabras, luego de un tiempo suficientemente largo, debería darse la evolución $\rho_{0}\left(x, t_{0}\right) \rightarrow \rho_{0}(x, t)=\rho_{1}(x)$.

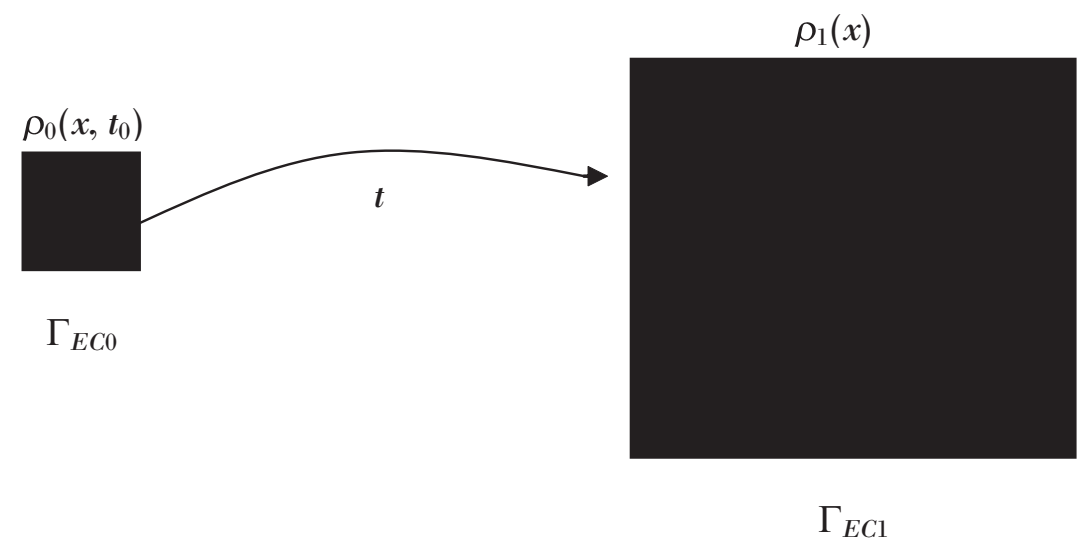


Sin embargo, tal situación no es posible. Los sistemas del ensemble inicial evolucionan según las leyes de la mecánica cumpliendo el teorema de Liouville de constancia de la medida en el espacio de las fases: la región $\Gamma_{E C 0}$ podrá deformarse y extenderse en la nueva región accesible del espacio de las fases pero, al mantener su volumen constante, no podrá cubrir de un modo efectivo la región $\Gamma_{E C 1}$ correspondiente al nuevo estado de equilibrio. En consecuencia, dada la validez del teorema de Liouville, la entropía de Gibbs $S_{G}$ se mantiene constante durante toda la evolución y, por lo tanto, no puede representar la entropía termodinámica $S$ regida por el segundo principio.

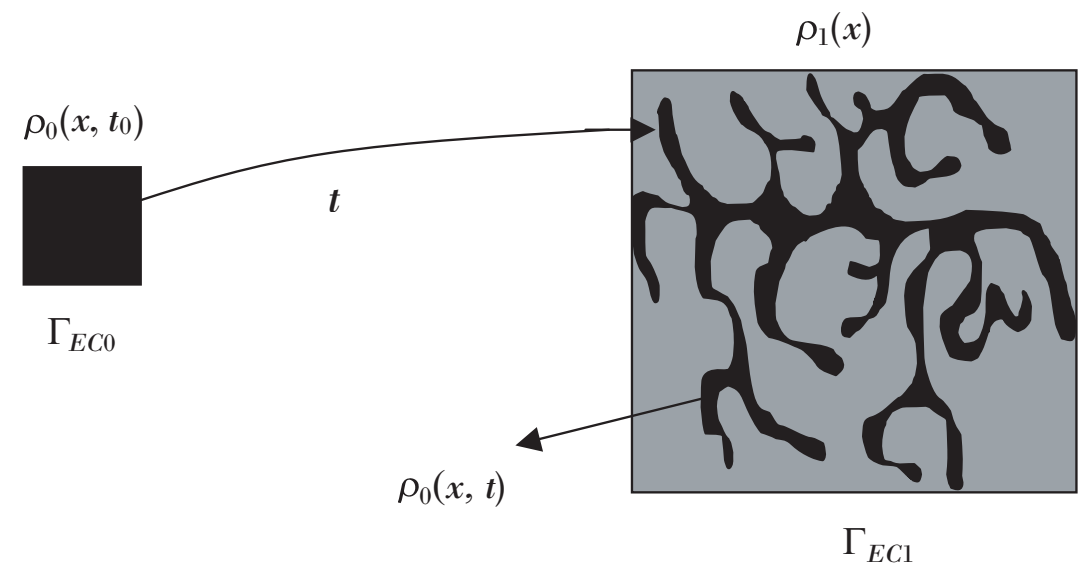

En el enfoque de Gibbs, lo que en realidad sucede es que la región inicial se ha distribuido y ramificado hasta el punto de cubrir de un modo aparentemente uniforme la región $\Gamma_{E C l}$ correspondiente al nuevo macroestado de equilibrio. A fin de dar cuenta de la creciente deformación de la región $\Gamma_{E C 0}$ inicial, puede definirse una entropía de grano grueso [coarse grain] $S_{c g}$ : divídase el espacio de las fases en celdas y asígnese una probabilidad $P_{i}$ a cada una de ellas - probabilidad de que el punto representativo del microestado del sistema se encuentre en la celda $i-; S_{c g}$ se define como:

$$
S_{c g}=-k \Sigma P_{i} \log P_{i}
$$

y puede esperarse que aumente a través de la evolución, a medida que la región inicial vaya ingresando en mayor cantidad de celdas. No obstante, si un observador "perfecto" describiera la evolución del 
sistema inicialmente fuera del equilibrio a través del comportamiento de su ensemble representativo, observaría la creciente distorsión y ramificación en el espacio de las fases de la región $\Gamma_{E C 0}$ correspondiente al macroestado inicial, pero podría comprobar la validez del teorema de Liouville: nunca se alcanza una distribución uniforme sobre la región $\Gamma_{E C l}$ asociada al nuevo macroestado de equilibrio, pues el volumen de la región inicial permanece invariante durante toda la evolución. En consecuencia, en el enfoque de Gibbs el aumento de la entropía termodinámica $S$ que enuncia el segundo principio sólo puede asociarse al aumento de entropía de grano grueso $S_{c g}$.

Desde la perspectiva de Gibbs, la paradoja de Loschmidt no constituye un problema en la medida en que el aumento de entropía sólo se produce en un sentido de grano grueso: la microevolución de los sistemas del ensemble es totalmente conservativo y reversible. Tampoco la objeción de la recurrencia introduce inconvenientes insalvables: el teorema de Poincaré afirma la recurrencia de las microevoluciones mecánicas de cada uno de los sistemas del ensemble; pero tales sistemas poseerán tiempos de recurrencia diferentes entre sí; por lo tanto, la validez del teorema de Poincaré para cada uno de los sistemas del ensemble es perfectamente compatible con el hecho de que el ensemble, como un todo, nunca vuelve a aproximarse a su situación inicial.

En principio, el enfoque de Gibbs no requiere la exclusión de condiciones microscópicas conflictivas: la descripción incluye la evolución de todos los sistemas del ensemble inicial. A su vez, tampoco requiere, en principio, un elevado número de grados de libertad del sistema bajo estudio: el comportamiento irreversible podría manifestarse en sistemas mecánicos simples, definidos por pocas variables de estado. Un aspecto importante de esta interpretación consiste en que, para producirse el aumento de la entropía de grano grueso $S_{c g}$, es necesario que la región correspondiente al estado inicial se deforme a través de la evolución. Como hemos señalado, para que esto ocurra, desde un punto de vista dinámico el sistema debe poseer la propiedad de mezcla; pero, a su vez, esto exige que el sistema sea ergódico, puesto que la mezcla es un grado de inestabilidad superior a la ergodicidad. ${ }^{11}$

\footnotetext{
${ }^{11}$ En la fundamentación de la mecánica estadística de Gibbs, las propiedades ergódicas cumplen un doble papel. Por un lado, mediante el teorema ergódico - Birkhoff_- en equilibrio se justifica la identificación entre el promedio en fase y el promedio temporal de una función de fase. Por otra parte, la ergodicidad es condición necesaria para el comportamiento irreversible del sistema. Este último
} 


\section{Boltzmann y Gibbs frente a frente}

La presentación de las secciones anteriores pone claramente de manifiesto los profundos desacuerdos entre los enfoques de Boltzmann y de Gibbs. En efecto, mientras que desde la perspectiva gibbsiana la irreversibilidad podría manifestarse en sistemas simples, definidos por pocas microvariables mecánicas, el enfoque de Boltzmann requiere que el sistema posea un elevado número de grados de libertad para manifestar un comportamiento irreversible. Los defensores del enfoque boltzmanniano insisten sobre este punto. Por ejemplo, según Lebowitz (1993, 1994), sólo la entropía de Boltzmann, definida en sistemas con un elevado número de microvariables de estado, captura la distinción entre las escalas microscópica y macroscópica. Desde una perspectiva similar, Bricmont (1995) insiste en la imposibilidad de brindar sentido físico a la distinción micro/macro en sistemas de pocos grados de libertad; además, sólo con un altísimo número de grados de libertad puede explicarse que nunca se observe a las macrovariables alejándose de su valor de equilibrio.

Por el contrario, la idea según la cual los sistemas dinámicos simples pueden manifestar un comportamiento irreversible fue explícitamente formulada por Gibbs (1902, p. VIII), y en la actualidad ha sido adoptada por autores como Hollinger y Zenzen (1982), quienes interpretan que la región del espacio de las fases que representa al sistema en $t=0$ queda exclusivamente definida por el error en la determinación empírica de las variables de estado mecánico. En su búsqueda de una irreversibilidad microscópica, los miembros de la escuela de Austin-Bruselas tampoco exigen alto número de grados de libertad sobre la base del análisis de modelos sencillos e idealizados. El ejemplo preferido por estos autores es la transformación del panadero, que consiste en un modelo matemático que posee las propiedades de un sistema $K$ : si bien se trata de un modelo sencillo, de dos grados de libertad, cualquier región finita del espacio de las fases evoluciona irreversiblemente hacia un estado final de equilibrio.

También respecto del papel que cumple la ergodicidad, los enfoques de Boltzmann y de Gibbs adoptan posiciones claramente divergentes. En el enfoque boltzmanniano, la ergodicidad no es condición necesaria para el comportamiento irreversible del sistema. La explicación gibbsiana de la irreversibilidad, por el contrario, depende esencialmente del carácter mezclador del sistema: si el sistema no es ergódico - y, por lo tanto, tampoco es mezclador- el esquema punto es el que se discute en el presente trabajo, y no el papel que desempeña la ergodicidad en equilibrio. 
teórico de Gibbs predice que no se producirá el aumento de la entropía de grano grueso asociada con la entropía macroscópica. Esto explica la razón por la cual quienes adoptan, en una u otra variante, la línea teórica de Gibbs siempre subrayan la necesidad de que la microestructura del sistema posea un alto grado de inestabilidad para que la irreversibilidad se manifieste. Uno de los primeros autores en señalar el papel esencial que cumple la inestabilidad dinámica para la irreversibilidad fue el físico ruso Krylov, al afirmar que "el proceso de mezcla es indispensable para que exista la relajación de los sistemas físicos, esto es, para hacer posible la existencia de una ley probabilística de la distribución de los estados que sea independiente del estado inicial" (Krylov 1979, p. 19).

La idea de mezcla, si bien carente por completo de definición formal, fue ya introducida por Gibbs en el capítulo XII de su Elementary Principles in Statistical Mechanics con su famosa analogía de la gota de tinta en un vaso de agua (Gibbs 1902, pp. 144-145). ${ }^{12}$ En su clásico texto, Tolman retoma explícitamente la misma idea:

esta disminución [de $k \Sigma P_{i} \log P_{i}$ ] resulta de la incapacidad de los puntos representativos del ensemble, originalmente presentes en alguna región particular pequeña pero finita $\delta q_{1} \ldots \delta p_{f}$ con densidad uniforme $\rho$, de moverse juntos como un todo compacto sin cambiar la "forma" de la extensión que ocupan. [...] Las diferentes densidades de grano fino $\rho$, dentro de una celda del espacio de las fases correspondiente a un rango $E$ a $E+\delta E$, finalmente se mezclan como para darnos una densidad de grano grueso $P$ aproximadamente uniforme dentro de ese rango. (Tolman 1938, pp. 177-178)

Es interesante notar cómo Gibbs y Tolman, aun sin contar con los elementos formales necesarios para relacionar ergodicidad y mezcla, consideran la propiedad de mezcla como un elemento indispensable para la evolución irreversible del sistema hacia el equilibrio.

Frente a la explicación gibbsiana de la irreversibilidad, los defensores del enfoque de Boltzmann adoptan una perspectiva probabilística según la cual un sistema evoluciona irreversiblemente desde sus macroestados menos probables hacia sus macroestados más probables:

\footnotetext{
${ }^{12} \mathrm{Si}$ mezclamos una gota de tinta negra en agua pura, el agua rápidamente se vuelve gris; sin embargo, un observador con los sentidos suficientemente desarrollados como para percibir las moléculas individuales nunca vería el color gris, pues podría seguir las trayectorias cada vez más deslocalizadas de las partículas de tinta inicialmente concentradas en una pequeña región del sistema. La idea de que el medio heterogéneo se ha convertido irreversiblemente en homogéneo es, entonces, resultado de describir el fenómeno desde una perspectiva de "grano grueso".
} 
esta explicación no requiere que el sistema sea ergódico. Por ejemplo, Lebowitz (1993) desacredita la entropía de Gibbs en la medida en que permanece constante durante la evolución del sistema. Presuponiendo la asimilación entre la entropía de Boltzmann y la entropía termodinámica, Callender (1999) formula una objeción análoga: mientras que la entropía de Gibbs continúa aumentando, el cambio en la entropía termodinámica cambia de signo durante la recurrencia. Desde una posición similar, Bricmont (1995) sostiene que todo intento por "forzar" el aumento de la entropía de Gibbs mediante particiones de grano grueso genera la incorrecta impresión de que la irreversibilidad sólo se debe a tales particiones y, por tanto, es un fenómeno subjetivo. La misma crítica expresa Callender cuando afirma que, en el enfoque gibbsiano, el comportamiento irreversible de la entropía "surge casi exclusivamente debido al grano grueso" (Callender 1999, p. 360), al que interpreta como una expresión de la imprecisión con la cual efectuamos mediciones sobre el sistema. A su vez, según Bricmont (1995), la ergodicidad no es condición ni necesaria ni suficiente para la irreversibilidad. No es condición suficiente puesto que hay sistemas ergódicos de pocos grados de libertad para los cuales no tiene sentido hablar de comportamiento irreversible. Pero la afirmación más fuerte es que la ergodicidad tampoco es condición necesaria, ya que existen evoluciones que, sin ser ergódicas, manifiestan un carácter inequívocamente irreversible. En efecto, el enfoque boltzmanniano sólo requiere que los puntos pertenecientes a la región definida por $F\left(t_{0}\right)$ den lugar a evoluciones que conduzcan a puntos pertenecientes a la región definida por $F_{\text {eq }}$; pero, para que esto se cumpla, no es necesario que el sistema sea ergódico. En palabras del autor:

considérese otra partición del espacio de las fases: se fija un conjunto de variables macroscópicas y se divide el espacio de las fases de acuerdo con los valores que adoptan dichas variables [...]. Cada elemento de la partición consiste en un conjunto de estados microscópicos que producen el mismo valor de las variables macroscópicas elegidas. Tales elementos de la partición tienen volúmenes muy diferentes [...]. De lejos, el mayor volumen corresponde a los valores de equilibrio de las variables macroscópicas. Por lo tanto, se necesita una noción mucho más débil que la ergodicidad. Todo lo que se requiere es que las configuraciones microscópicas evolucionen en el espacio de las fases hacia aquellas regiones donde las variables macroscópicas relevantes toman sus valores de equilibrio. (Bricmont 1995, p. 21) 
Lebowitz se refiere a los procesos irreversibles en un sentido análogo: "las características esenciales de la evolución no dependen de propiedades dinámicas específicas, como la positividad de los exponentes de Lyapounov, la ergodicidad o la mezcla" (Lebowitz 1993, p. 32). En una línea similar de crítica al enfoque de Gibbs, Earman y Rédei señalan que muchos de los sistemas típicos estudiados en mecánica estadística no son siquiera ergódicos (Earman y Rédei 1996, p. 70). Un argumento particularmente fuerte en favor del enfoque de Boltzmann es el que esgrime Bricmont al presentar el modelo Kac como un ejemplo claro y sencillo donde se manifiesta un comportamiento irreversible sin ergodicidad.

Como puede observarse, los argumentos que entran en juego en el debate no se limitan a consideraciones conceptuales y teóricas. Los participantes de la discusión presentan ejemplos concretos que respaldan sus respectivas posiciones. El paso siguiente será, entonces, analizar dichos ejemplos a fin de comprender en profundidad el problema bajo estudio.

\section{Irreversibilidad con pocos grados de libertad: la transformación del panadero}

La transformación del panadero es el ejemplo preferido por la escuela de Austin-Bruselas para ilustrar el modo en que la irreversibilidad surge en sistemas altamente inestables: ${ }^{13}$ se trata de una transformación discreta en un espacio de las fases de dos variables, $x$ e $y$, tales que $0 \leq x \leq 1$ y $0 \leq y \leq 1$; la transformación queda definida por las siguientes ecuaciones:

\footnotetext{
${ }^{13}$ En su versión simplificada, esta transformación es algo así como la versión cubista de la gota de Gibbs. Supóngase que existe un panadero que decide amasar su bollo de la siguiente manera: en primer lugar, estira la masa de modo que su ancho se duplique y su espesor se reduzca a la mitad; luego separa la mitad derecha de la masa y la coloca exactamente sobre la otra mitad, y obtiene un bollo de iguales dimensiones que el original; a continuación repite indefinidamente la misma operación. Si se considera un punto preciso de la masa, en teoría puede determinarse qué posición ocupará luego de $n$ operaciones; sin embargo, si se tiñe una pequeña porción de masa y se inicia el proceso de amasado, la mancha irá fracturándose progresivamente hasta que, finalmente, el color se diluya repartiéndose en toda la masa. No importa qué tan pequeña sea la mancha original: llegará el momento en que se fragmentará y su color inevitablemente se difundirá luego de un número suficiente de iteraciones. Pero, al igual que en el caso de la gota de Gibbs, esta presentación simplificada de la transformación del panadero no es más que una analogía en el espacio físico de lo que sucede en el espacio de las fases.
} 


$$
\left\{\begin{array}{lll}
x^{\prime}=2 x & y^{\prime}=y / 2 & \text { para } 0 \leq x<\frac{1}{2} \\
x^{\prime}=2 x-1 & y^{\prime}=(y+1) / 2 & \text { para } \frac{1}{2} \leq x \leq 1
\end{array}\right.
$$

y corresponde a una evolución que ocurre a intervalos regulares; por ejemplo, cada segundo. En cada iteración, la región cuadrada original se estira al doble de su tamaño en la dirección horizontal y se contrae a la mitad en la dirección vertical; luego, la mitad derecha del rectángulo resultante se "corta" y se ubica sobre la mitad izquierda:

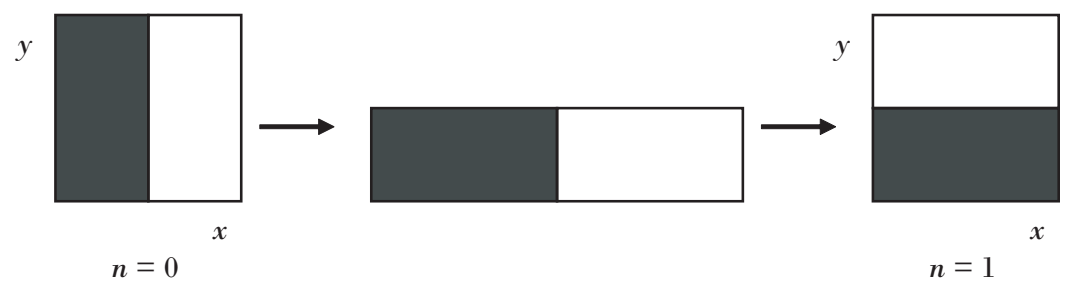

La microevolución resultante es totalmente determinista puesto que, a partir de cada punto, conduce a un nuevo punto unívocamente determinado. Las ecuaciones que definen la transformación son invariantes frente a la inversión temporal: si la transformación se aplica en sentido inverso, vuelve a obtenerse la distribución original. En efecto, si las ecuaciones son resueltas para $x$ e $y$ se obtiene:

$$
\left\{\begin{array}{lll}
x=x^{\prime} / 2 & y=2 y^{\prime} & \text { para } 0 \leq y<\frac{1}{2} \\
x=\left(x^{\prime}+1\right) / 2 & y=2 y^{\prime}-1 & \text { para } \frac{1}{2} \leq y \leq 1
\end{array}\right.
$$

Estas últimas son idénticas a la versión invertida en el tiempo de las ecuaciones originales, dado que $x$ e $y$ se han intercambiado; tal intercambio es análogo a la inversión de las velocidades manteniendo invariantes las coordenadas espaciales, cuando se invierte el signo de la variable $t$ en las ecuaciones de la mecánica clásica. Además, se trata de una evolución conservativa: se cumple el teorema de Liouville de conservación de la medida en el espacio de las fases, medida que aquí corresponde a una superficie ( $c f r$. Nicolis y Prigogine 1989).

Si se toma un punto cualquiera como condición inicial, la secuencia de puntos que resulta de la aplicación sucesiva de la transformación es totalmente determinista y reversible. Sin embargo, si las condiciones iniciales quedan representadas por una región de superficie finita, tras un número suficiente de iteraciones tal región acabará fragmentándose por la operación de "corte" del rectángulo, sin importar su 
ubicación inicial ni qué tan pequeña sea su superficie; los fragmentos, a su vez, sufrirán el mismo tipo de fragmentación:

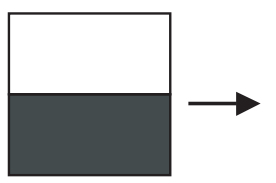

$n=0$

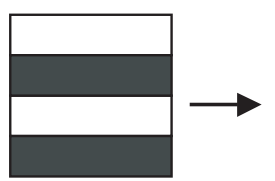

$n=1$

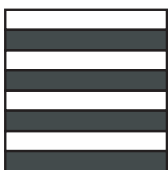

$n=2$

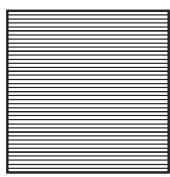

$n \rightarrow \infty$

Es decir, con el transcurso del tiempo cualquier región finita quedará particionada en franjas cada vez más delgadas, hasta parecer finalmente distribuida de un modo uniforme: los puntos pertenecientes a la región inicial cubrirán densamente la totalidad del espacio de las fases de modo tal que, en cualquier zona, por pequeña que sea, se encontrarán puntos resultantes de la transformación de la región original. Estas características indican que cualquier evolución es altamente inestable: a partir de dos puntos cualesquiera arbitrariamente próximos se originan trayectorias que divergen exponencialmente con el tiempo. ${ }^{14}$ Sobre la base de tal divergencia exponencial puede demostrarse que se trata de un sistema $K$, cuya descripción de grano grueso en términos de macroeventos — celdas en el espacio de las fasesconduce a una macroevolución indeterminista e irreversible. Pero, como fue señalado, la propiedad de ser un sistema $K$ es más fuerte que la ergodicidad y, por tanto, la implica.

En resumen, la microdinámica descrita por la transformación del panadero es determinista, reversible, conservativa y altamente inestable. Sin embargo, si las condiciones iniciales no se representan mediante un punto sino mediante una región, luego de un tiempo suficientemente largo, los puntos correspondientes a la región inicial se encontrarán en cualquier parte del espacio de las fases con igual probabilidad (Prigogine y Stengers 1991). Por lo tanto, en la descripción de grano grueso aparecen "aspectos estadísticos irreductibles [... ] cuando la evolución de un punto es reversible y determinista, la descripción de una región, no importa lo pequeña que sea,

${ }^{14}$ La divergencia exponencial es la característica definitoria del comportamiento caótico. Por lo tanto, es posible calcular el exponente de Lyapounov correspondiente a este tipo de evoluciones. La distancia $\delta_{x}(0)$ que separa dos puntos cualesquiera según el eje $x$ se duplica en cada iteración; en consecuencia, luego de $n$ iteraciones la distancia será $\delta_{x}(n)=\delta_{x}(0) 2^{n}=\delta_{x}(0) e^{n l n 2}$; si $n$ desempeña el papel del tiempo de evolución, se obtienen los valores del exponente de Lyapounov, $h=\ln 2$, y del tiempo de Lyapounov, $\tau=1 / h=1 / \ln 2$. Dado que se trata de un sistema conservativo, existirá también un segundo exponente de Lyapounov según el eje $y$ de valor negativo $h^{\prime}=-\ln 2$. 
es básicamente estadística, sólo podemos hablar de la probabilidad de que un sistema cuya condición inicial corresponde a una región, siga tal o cual tipo de trayectoria" (Prigogine y Stengers 1990, p. 275). Desde la perspectiva de los defensores del enfoque de Gibbs, estas características de la transformación "permiten establecer una genuina irreversibilidad" (Nicolis y Prigogine 1989, p. 203). En efecto, si a la manera de Gibbs, la región inicial se describe mediante una función de distribución $\rho_{0}(x, t)$, ésta evolucionará cumpliendo el teorema de Liouville de constancia de volumen en fase. Pero dado que, luego de un tiempo suficientemente largo, los puntos correspondientes a la región inicial se habrán distribuido de un modo prácticamente uniforme por todo el espacio de las fases, la entropía de grano grueso $\mathrm{S}_{c g}$ aumentará a través de la evolución, en concordancia con la explicación gibbsiana de la irreversibilidad. Sobre esta base, Driebe concluye: "los procesos irreversibles son bien observados en sistemas de pocos grados de libertad, como las transformaciones del panadero y del multipanadero. Por lo tanto, muchos grados de libertad no es condición para el comportamiento irreversible" (Driebe 1994, p. 15).

\section{Irreversibilidad sin ergodicidad: el modelo Kac}

En el modelo Kac (Kac 1959) se manifiesta un comportamiento irreversible que depende esencialmente de las restricciones boltzmannianas, pero que no requiere las condiciones que impone el enfoque de Gibbs. En efecto, en tal modelo las microevoluciones son perfectamente deterministas, recurrentes y reversibles; no obstante, si el número de grados de libertad es lo suficientemente elevado, pueden definirse macrovariables que tienden irreversiblemente a un macroestado de equilibrio en una escala de tiempo enormemente inferior al tiempo de recurrencia; a su vez, pueden identificarse fácilmente los microestados iniciales excepcionales a partir de los cuales tal evolución irreversible no se produce. No obstante, el modelo Kac no es ergódico, en total desacuerdo con la condición de mezcla que exige el enfoque de Gibbs; por lo tanto, podría existir irreversibilidad sin ergodicidad.

Para construir el modelo Kac, se señalan sobre un círculo $n$ puntos equidistantes de modo de definir $n$ segmentos iguales: $m$ segmentos se marcan de algún modo y forman el conjunto $S$; el conjunto complementario de los $n-m$ segmentos no marcados será $S^{\prime}$. En cada uno de los puntos se ubica una bola que podrá ser blanca $(w)$ o negra $(b)$. Durante cada $\Delta t$ elemental, cada bola se mueve en sen- 
tido antihorario hasta el punto contiguo, cumpliendo las siguientes condiciones:

- Si la bola cruza un segmento perteneciente a $S$, cambia de color.

- Si la bola cruza un segmento perteneciente a $S^{\prime}$, no cambia de color.

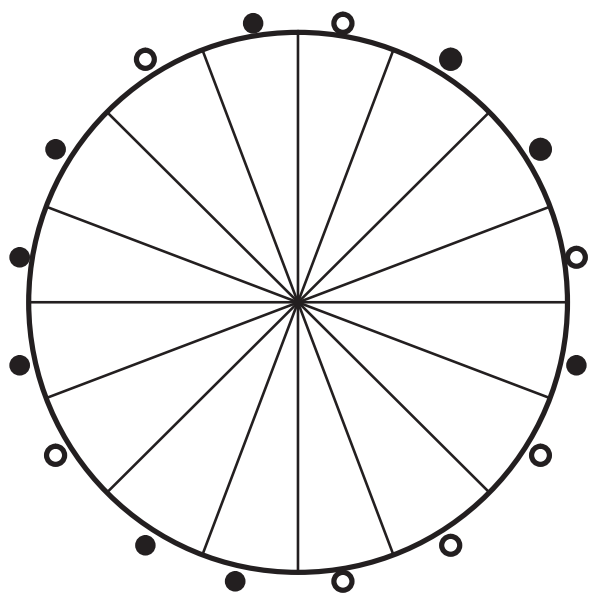

Bricmont subraya la analogía con el caso mecánico. El microestado de cada una de las bolas queda definido por su posición y por su color: el color cumple el papel de la velocidad - discreta - puesto que cambia cuando la bola colisiona con un "dispersor" [scatterer] fijo, esto es, un segmento perteneciente a $S$. La característica que simplifica el modelo es que la velocidad-color no afecta el movimiento. La microdinámica del sistema viene dada por el movimiento antihorario más los cambios de color. La microevolución:

- Es determinista y reversible: si luego de $k$ intervalos se invierte la orientación del movimiento, el sistema vuelve a su microestado inicial luego de $k$ pasos.

- Es periódica: luego de $2 n$ pasos, cada segmento ha sido atravesado dos veces por cada bola y, por tanto, cada bola ha vuelto a su posición y su color original.

Dada esta descripción microscópica del sistema, se definen ahora como macrovariables el número de bolas blancas $N_{w}(t)$ y el número de bolas negras $N_{b}(t)$ en cada instante. El problema es determinar, dada una distribución inicial arbitraria de colores, qué sucede 
con $N_{w}(t)$ y con $N_{b}(t)$ luego de un gran número de movimientos. Kac calcula, para cada configuración $S / S^{\prime}$ de segmentos, la curva $\left(N_{w}(t)-N_{b}(t)\right) / n$ como función del tiempo; el resultado es que, para $n$ suficientemente elevado, para cada $t$, la enorme mayoría de tales curvas se aproxima a $(1-2 m / n)^{t}$. Si además se cumple que $2 m<n$, entonces para tiempos suficientemente grandes $(1-2 m / n)^{t}$ tiende a cero y, por tanto, $N_{w}(t)-N_{b}(t)$ tiende a cero, esto es, el sistema tiende a la equipartición entre bolas blancas y bolas negras. Esta evolución irreversible de las macrovariables se obtiene a pesar de que el sistema es periódico de periodo $2 n$ : si $n$ es suficientemente elevado, el macroestado de equilibrio - equipartición - se obtiene para tiempos inferiores en varios órdenes de magnitud al tiempo de recurrencia $2 n$ (Kac sugiere pensar en $n$ del orden de $10^{23}$ y $t$ del orden de $10^{6}$ ).

Pero la cuestión central aquí es que el modelo Kac, si bien manifiesta la evolución irreversible de las macrovariables $N_{w}(t)$ y $N_{b}(t)$, no es ergódico. En efecto, dado que se trata de un sistema discreto, el espacio de las fases $\Gamma$ del modelo Kac contiene $2^{n} . n$ ! microestados posibles: las $n$ ! permutaciones de posición de las $n$ bolas consideradas distinguibles, por las $2^{n}$ combinaciones posibles de los dos colores en las $n$ bolas. Pero respecto de las macrovariables $N_{w}(t)$ y $N_{b}(t)$, número total de bolas blancas y de bolas negras respectivamente, en $t$, no es relevante la posición de cada una de las bolas sino sólo su color; por lo tanto, la ergodicidad y la mezcla relevantes deben definirse sobre el subespacio-color $\Gamma_{C}$ que contiene sólo las $2^{n}$ combinaciones posibles de los dos colores en las $n$ bolas. Pero, dado que el sistema es periódico de periodo $2 n$, ninguna trayectoria puede pasar por más de $2 n$ microestados-color: cada trayectoria sólo pasa por una pequeña región del subespacio-color $\Gamma_{C}$. Sobre la base de este ejemplo, Bricmont concluye que "esto ilustra claramente el hecho de que la ergodicidad no es necesaria para la irreversibilidad" (Bricmont 1995, p. 41).

\section{Las nociones de equilibrio e irreversibilidad}

El disenso entre los enfoques de Boltzmann y de Gibbs queda así claramente planteado. Para quienes adoptan la línea gibbsiana, es indispensable que un sistema sea al menos mezclador - y, por tanto, ergódico - para manifestar un comportamiento irreversible; sin embargo, el elevado número de grados de libertad no es condición necesaria para la irreversibilidad, tal como lo demuestran ciertos ejemplos definidos en espacios de las fases de muy pocas dimensio- 
nes. Los defensores del enfoque de Boltzmann, por su parte, exigen un elevado número de grados de libertad, pero sostienen que la ergodicidad no es condición necesaria para la irreversibilidad e incluso esgrimen ejemplos de sistemas dinámicos no ergódicos que describen evoluciones irreversibles hacia el equilibrio. Esta situación es particularmente paradójica en la medida en que los enfoques de Boltzmann y de Gibbs son los dos marcos teóricos tradicionalmente aceptados en mecánica estadística. No obstante, el problema comienza a disiparse cuando se analizan los conceptos de equilibrio e irreversibilidad en ambos enfoques.

Suele afirmarse que las entropías de Gibbs y de Boltzmann coinciden con la entropía termodinámica en el equilibrio: "en equilibrio [la entropía de Gibbs] coincide con las entropías de Boltzmann y de Clausius (a menos de términos que son despreciables cuando el número de partículas es alto), y todo está bien" (Bricmont 1995, p. 188). Esta opinión parece suponer que no hay dificultad alguna en el caso de equilibrio, y que los problemas de fundamentación de la mecánica estadística se concentran alrededor de la diferencia entre los enfoques de Boltzmann y de Gibbs en sus explicaciones de la evolución hacia el equilibrio. Sin embargo, tal supuesto pasa por alto un hecho central: la diferencia entre los modos en que los dos enfoques dan cuenta de una dinámica irreversible encuentra su origen en una importante diferencia entre los propios conceptos de equilibrio formulados en ambos marcos teóricos.

Como fue señalado, en el enfoque de Gibbs el equilibrio se identifica con el equilibrio estadístico, asociado a la distribución microcanónica que adopta un valor constante en toda la región $\Gamma_{E C}$ del espacio de las fases accesible al sistema, esto es, definida por su energía $E$ y los vínculos $C_{k}$ impuestos externamente. Por lo tanto, el equilibrio gibbsiano queda representado por una distribución $\rho(x)$ que adopta valores no nulos en toda la región accesible $\Gamma_{E C}$. En el enfoque de Boltzmann, por el contrario, el equilibrio se asimila al equilibrio probabilístico, es decir, al macroestado $M_{e q}$ con mayor número de microestados compatibles. A su vez, el macroestado de equilibrio $M_{e q}$ queda representado en el espacio de las fases por la región $\Gamma_{e q}=\Gamma\left(F_{e q}\right)$, en la cual la función de fase $f(x)$ adopta el valor de equilibrio $F_{e q}$ de la variable macroscópica. Pero si recordamos que la variable macroscópica $F(t)$ introduce una partición sobre la región accesible $\Gamma_{E C}$ y que $\Gamma_{e q}$ es sólo un elemento de dicha partición, queda claro que $\Gamma_{e q}$ no puede asimilarse a $\Gamma_{E C}$, sino que $\Gamma_{e q} \subset \Gamma_{E C}$. En otras palabras, el equilibrio en el sentido de Boltzmann queda 
representado por una región del espacio de las fases incluida en la región correspondiente al equilibrio gibbsiano.

Por otra parte, el equilibrio gibbsiano queda definido por las propiedades más generales del sistema, esto es, su energía y los vínculos impuestos externamente: para un sistema dado, con su energía y sus vínculos, el equilibrio representado por la distribución microcanónica es único. El equilibrio boltzmanniano, por el contrario, aun en un mismo sistema caracterizado por su región accesible $\Gamma_{E C}$, es relativo a la variable macroscópica $F$ considerada. En efecto, otra variable macroscópica $F^{\prime}$ puede introducir una partición diferente en la región accesible del espacio de las fases, de modo tal que el nuevo estado de equilibrio $M_{e q^{\prime}}$ quede representado por una región $\Gamma_{e q^{\prime}} \neq \Gamma_{e q}$.

Si bien las diferencias entre los dos conceptos de equilibrio han sido indicadas por algunos autores ( $c f r$. Lavis 2005), no suele señalarse explícitamente que tal diferencia conduzca directamente a dos nociones distintas de irreversibilidad. En efecto, en el enfoque de Gibbs, la evolución de un sistema es irreversible si tiende al equilibrio estadístico. En este caso, el equilibrio es un estado atractor de la descripción de grano grueso, es decir, un estado al que se tiende para tiempo tendiendo a infinito y del cual el sistema ya no puede escapar. Es precisamente la propiedad de mezcla, en cuanto propiedad asintótica, la que asegura la permanente distorsión y ramificación de la región correspondiente a la distribución inicial, que conducirán finalmente al sistema al estado atractor. En el enfoque boltzmanniano, en cambio, una evolución es irreversible si alcanza el equilibrio probabilístico, es decir, si el punto representativo del microestado del sistema en el espacio de las fases ingresa en la región de equilibrio $\Gamma_{e q}$. Tal tipo de evolución no requiere introducir el límite para tiempo infinito, sino que puede ocurrir en un tiempo finito. Pero el equilibrio no es aquí un estado atractor, puesto que el sistema puede abandonar el estado de equilibrio en un tiempo suficientemente largo, del orden del tiempo de recurrencia de Poincaré. En efecto, luego del tiempo de recurrencia el sistema retornará indefinidamente cerca de su microestado inicial correspondiente al macroestado inicial de no equilibrio.

A su vez, las dos nociones de equilibrio conducen a diferentes modos de concebir las posibles fluctuaciones que sufre el sistema como consecuencia de su dinámica microscópica. En el enfoque de Gibbs, la entropía de Gibbs $S_{G}$ adopta un valor constante en el equilibrio y, en consecuencia, no fluctúa; las fluctuaciones quedan confinadas al nivel microscópico subyacente. En el enfoque boltzmanniano, por el 
contrario, el punto representativo del microestado del sistema puede escapar temporalmente de la región de equilibrio y pasar a macroestados de menor entropía. Por lo tanto, la entropía de Boltzmann $S_{B}$ puede fluctuar aun luego de que el sistema ha alcanzado el equilibrio, y tiene sentido afirmar que el sistema experimenta fluctuaciones fuera del equilibrio. El punto central que debe justificarse en este enfoque consiste en que, en la gran mayoría de los casos, $S_{B}$ se comporta de un modo termodinámico, esto es, experimenta frecuentes fluctuaciones pequeñas pero sólo excepcionales fluctuaciones amplias respecto de su valor de equilibrio.

Cuando se advierte la diferencia entre los conceptos de equilibrio e irreversibilidad que manejan ambos enfoques, el debate acerca de la irreversibilidad, tal como se suele plantear, se disuelve. Ya no se trata de determinar cuáles son las condiciones necesarias para la irreversibilidad, como si nos estuviéramos refiriendo a un concepto único, sino de constatar que el enfoque de Gibbs da cuenta de un tipo de irreversibilidad diferente de la irreversibilidad explicada por la perspectiva de Boltzmann. Pierde sentido, entonces, todo intento de desacreditar la explicación de las evoluciones irreversibles que brinda cada uno de los enfoques desde la otra perspectiva. Ambas posiciones resultan correctas sobre la base de sus propias definiciones de equilibrio e irreversibilidad. A su vez, no existe contradicción alguna en sostener que la ergodicidad es condición necesaria para la irreversibilidad gibbsiana, pero no para la boltzmanniana, o que un elevado número de grados de libertad es condición necesaria para la irreversibilidad boltzmanniana, pero no para la gibbsiana.

Una vez que los dos conceptos de irreversibilidad han sido claramente elucidados, los modelos que los defensores de cada enfoque presentan como contraejemplos para el otro enfoque ya no cumplen el papel que originalmente se pretendía adjudicarles. El caso del modelo Kac, esgrimido por los defensores de la línea boltzmanniana, deja de representar un desafío para el enfoque de Gibbs. En efecto, la evolución de las macrovariables del sistema Kac es irreversible en el sentido de Boltzmann, pero es reversible en el sentido de Gibbs, puesto que el estado de equipartición entre bolas blancas y bolas negras, que se alcanza en un tiempo finito, no es un estado atractor y, por lo tanto, el sistema volverá a su estado inicial después de un número suficiente de movimientos. En cuanto a la transformación del panadero, utilizada por quienes adoptan la perspectiva de Gibbs como ejemplo de irreversibilidad en sistemas de pocos grados de libertad, sin duda representa un caso de irreversibilidad gibbsiana. No obstante, en este caso no puede hablarse de irreversibilidad en el 
sentido de Boltzmann pues la ausencia de un elevado número de grados de libertad impide definir variables macroscópicas cuyos estados de equilibrio macroscópicos pueden determinarse significativamente.

Si bien de este modo se disuelve la aparente contradicción planteada por el desacuerdo entre los enfoques de Boltzmann y de Gibbs, la conclusión anterior conduce a un nuevo problema: ¿̨cuál de los dos conceptos de equilibrio y, por lo tanto, de irreversibilidad es el físicamente relevante? Podría argumentarse que, dado el altísimo número de grados de libertad de los sistemas estudiados en mecánica estadística, el tiempo de recurrencia es tan grande que resulta inobservable en la práctica y, en consecuencia, el enfoque boltzmanniano es el que da cuenta de la irreversibilidad efectivamente observable. Sin embargo, esta posición deja sin explicar el éxito predictivo del enfoque de Gibbs, el cual, dada su generalidad, se ha constituido en la herramienta teórica estándar de la mecánica estadística, no sólo clásica sino también cuántica. En el próximo apartado abordaremos este nuevo problema, argumentando que, en las condiciones físicas más usuales, ambos enfoques pueden compatibilizarse de un modo satisfactorio.

\section{Compatibilización de ambos enfoques}

Como ya fue señalado, los enfoques de Boltzmann y de Gibbs dan cuenta de dos formas distintas de irreversibilidad, en la medida en que trabajan con diferentes conceptos de equilibrio: el equilibrio gibbsiano representado por una distribución cuyo soporte se encuentra confinado en la región $\Gamma_{E C}$ del espacio de las fases, y el equilibrio boltzmanniano representado por la región $\Gamma_{e q} \subset \Gamma_{E C}$. Dado que la medida $\mu$ de una región del espacio de las fases puede asociarse a su volumen, la relación de inclusión entre ambas regiones asegura que $\mu\left(\Gamma_{E C}\right)>\mu\left(\Gamma_{e q}\right)$. Es posible que esta disparidad entre las dos regiones no sea muy grande en sistemas de pocos grados de libertad; pero, a medida que el número $N$ de grados de libertad aumenta - y, por lo tanto, el número de dimensiones del espacio de las fases también aumenta-, el macroestado de equilibrio boltzmanniano $M_{e q}$ ocupa una región $\Gamma_{e q}$ relativamente mayor en la región accesible $\Gamma_{E C}$, de modo tal que la distinción entre las medidas de $\Gamma_{e q}$ y $\Gamma_{E C}$ tiende a disminuir: la relación entre ambas medidas, $\mu\left(\Gamma_{e q}\right) / \mu\left(\Gamma_{E C}\right)$, tiende a uno en el límite termodinámico $N \rightarrow \infty .^{15}$ En otras palabras, cuando se trabaja con sistemas con un altísimo número de grados

\footnotetext{
${ }^{15}$ Esto puede demostrarse en el caso sencillo de un par de cajas idénticas que contienen $m$ y $n-m$ bolas distinguibles, respectivamente. Las bolas pueden distribuirse en un número de formas dado por: $C(n, m)=n ! /(m !(n-m) !$. En el caso
} 
de libertad, los conceptos de equilibrio boltzmanniano y gibbsiano se aproximan lo suficiente como para ser considerados equivalentes desde el punto de vista práctico, aunque no coinciden desde un punto de vista conceptual.

En consecuencia, la afirmación según la cual las entropías de Boltzmann y de Gibbs coinciden en el equilibrio no es estrictamente correcta. En efecto, en equilibrio, la entropía de Gibbs $S_{G}$ se calcula mediante la distribución microcanónica, cuyo soporte se encuentra confinado en toda la región accesible $\Gamma_{E C}$, mientras que la entropía de Boltzmann $S_{B}$ se define sobre la región $\Gamma_{e q}$ correspondiente al macroestado de equilibrio. Esto significa que, cuando el número $N$ de grados de libertad del sistema es finito, ambas entropías tienen diferentes valores en los correspondientes estados de equilibrio. Sólo en el límite termodinámico $N \rightarrow \infty$, cuando ambas regiones $\Gamma_{E C}$ y $\Gamma_{e q}$ tienden a identificarse $-\mathrm{y}$, consecuentemente, también ambos equilibrioslas entropías de Boltzmann y de Gibbs tienden a coincidir.

Por otra parte, el tiempo de recurrencia de Poincaré también aumenta enormemente con el aumento del número $N$ de grados de libertad del sistema, e incluso tiende a infinito en el límite termodinámico $N \rightarrow \infty$ (para resultados cuantitativos, cfr. Blaise et al. 1994, Gaioli y García-Alvarez 1999). Dado este hecho dinámico y la relación entre probabilidad y volumen en el espacio de las fases, en sistemas con un altísimo número de grados de libertad, la probabilidad de que el sistema abandone el estado de equilibrio boltzmanniano es tan ínfima que puede despreciarse sin errores significativos. En otras palabras, si bien el equilibrio boltzmanniano no es un estado atractor en un sentido teórico, en sistemas con un elevadísimo número de grados de libertad se comporta como tal a todo efecto práctico.

Si los conceptos de equilibrio $-\mathrm{y}$, en consecuencia, de irreversibilidad - en los enfoques de Boltzmann y de Gibbs se aproximan indefinidamente con el aumento del número de grados de libertad del sistema, ¿significa esto que en el límite termodinámico ambos enfoques tienden a identificarse bajo un único marco teórico? La respuesta a esta pregunta es negativa pues, aun cuando ambos conceptos de equilibrio puedan aproximarse tanto como se quiera, los enfoques de Boltzmann y de Gibbs dan cuenta de la evolución irreversible hacia el equilibrio de maneras diferentes. En particular, la explicación gibbsiana de la irreversibilidad requiere la propiedad de mezcla - y, en consecuencia, de ergodicidad-, y tal condición no desaparece

$m=n / 2$, este número se hace máximo e igual a $C(n, n / 2)=n ! /((n / 2) !)^{2}$. El cociente $C(n, m) / \mathrm{C}(n, n / 2)$ tiende a cero para $n \rightarrow \infty$, para cualquier valor de $m$. 
como tal con el aumento del número de grados de libertad del sistema. Por lo tanto, no se trata de dos enfoques teóricos que tiendan a identificarse en un cierto límite, sino de dos perspectivas diferentes que se solapan bajo ciertas condiciones definidas, tal como indica el siguiente esquema:

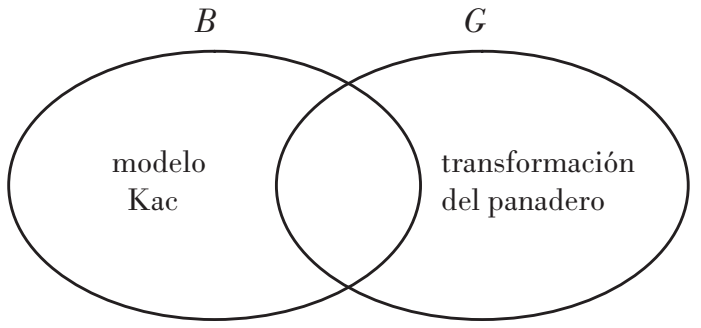

El óvalo de la izquierda representa el conjunto $B$ de sistemas para los cuales la explicación de la irreversibilidad suministrada por el enfoque de Boltzmann brinda resultados adecuados; se trata, entonces, de sistemas con un alto número de grados de libertad. El óvalo de la derecha representa el conjunto $G$ de sistemas cuyo comportamiento irreversible puede ser explicado por la perspectiva de Gibbs; en este caso se trata de sistemas mezcladores y, por tanto, ergódicos.

El esquema pone de manifiesto claramente la diferencia entre los dos enfoques. El conjunto $B-G$ está compuesto por los sistemas cuya irreversibilidad es exclusivamente del tipo boltzmanniano. A este conjunto pertenece el modelo Kac con su elevado número de grados de libertad, pero donde la ausencia de la propiedad de mezcla impide concebir la evolución como un caso de irreversibilidad gibbsiana. A su vez, el conjunto $G-B$ está compuesto por los sistemas cuya irreversibilidad es exclusivamente del tipo gibbsiano. A este conjunto pertenece la transformación del panadero, que posee la propiedad de mezcla pero no permite definir variables macroscópicas a la manera de Boltzmann debido a sus pocos grados de libertad.

La intersección $B \cap G$ entre ambos conjuntos del esquema viene dada por los sistemas mezcladores con un elevado número de grados de libertad: en estos casos, tanto los conceptos de equilibrio como los de irreversibilidad de ambos enfoques tienden a asimilarse. Además, dado que se trata de sistemas mezcladores con un elevado número de grados de libertad, ambos enfoques brindan resultados correctos en sus respectivas explicaciones de la irreversibilidad. En consecuencia, en el caso de los sistemas pertenecientes a la intersección $B \cap G$, tanto el enfoque de Boltzmann como el de Gibbs 
pueden ser utilizados de un modo satisfactorio para dar cuenta de la evolución irreversible hacia el equilibrio.

No obstante, aún es necesario preguntarse por la relevancia física del caso particular en el que ambos enfoques tienden a coincidir. La respuesta se obtiene teniendo en cuenta que, si bien el conjunto $B \cap G$ constituye una situación particular desde un punto de vista conceptual, en la práctica científica los sistemas tradicionalmente estudiados en mecánica estadística suelen cumplir las dos condiciones - mezcla y elevado número de grados de libertad- y, en consecuencia, corresponden a esta situación. En efecto, el ejemplo paradigmático de sistema al que se aplican las herramientas teóricas de la mecánica estadística es el de un gas, compuesto aproximadamente por $6.10^{20}$ partículas por mol, cada una de las cuales aporta al menos seis dimensiones al espacio de las fases. Por otra parte, las investigaciones en teoría ergódica ponen de manifiesto el carácter ubicuo de la inestabilidad en los modelos de sistemas físicos, aun aquellos fuertemente idealizados. Si bien los sistemas dinámicos sencillos pueden poseer las propiedades ergódicas más fuertes, tales propiedades suelen presentarse con mayor frecuencia en sistemas con un elevado número de grados de libertad. Por lo tanto, el caso que resulta particular desde un punto de vista conceptual, se convierte en genérico cuando se lo considera desde la perspectiva de la práctica efectiva de la física.

\section{La diferencia conceptual entre ambos enfoques}

Como hemos señalado, según una opinión difundida, las divergencias entre los enfoques de Boltzmann y de Gibbs se refieren a la explicación de la irreversibilidad macroscópica, esto es, a la cuestión dinámica de la evolución hacia el equilibrio; es en este ámbito dinámico donde se presentan los disensos acerca del papel de la ergodicidad y del elevado número de grados de libertad, problemas en los cuales hemos concentrado la atención en las secciones previas. Sin embargo, tal como hemos visto, las divergencias respecto de la cuestión dinámica tienen su origen en la diferencia entre los conceptos de equilibrio gibbsiano y boltzmanniano. A su vez, tal diferencia es la manifestación de los distintos supuestos teóricos básicos sobre los cuales se construyen ambos enfoques.

En primer lugar, los dos enfoques se distinguen por el modo en que la probabilidad se incorpora a la descripción. En el enfoque de Boltzmann, la probabilidad se concibe en términos combinatorios: la probabilidad de cada macroestado viene dada por el número de microestados mecánicos compatibles con dicho macroestado, bajo el 
supuesto de que todos los microestados son igualmente probables $a$ priori. En el enfoque de Gibbs, en cambio, la probabilidad ingresa a través del concepto de ensemble; la densidad de distribución $\rho$ es la magnitud que define el estado del ensemble. Por lo tanto, mientras que en el enfoque boltzmanniano el equilibrio y la entropía son propiedades de un sistema único, en el marco teórico gibbsiano resultan propiedades del ensemble. Ahora bien, dado que el sistema cuya descripción estadística se pretende obtener es un sistema único, el enfoque gibbsiano se enfrenta al desafío de justificar la introducción de los ensembles. Originalmente, el propio Gibbs adoptó una actitud pragmática: puesto que el valor de una teoría debe estimarse únicamente por su capacidad de dar cuenta de los resultados empíricos, no es necesario que cada uno de sus términos posea una referencia en la realidad; en consecuencia, la utilización de ensembles se justifica por su éxito en el cálculo de los valores observables de las variables macroscópicas (cfr. Guttmann 1999). No obstante, muchos continuadores del enfoque gibbsiano abandonaron esta actitud pragmática; por ejemplo, en su influyente libro, Tolman (1938) considera el ensemble representativo del sistema bajo estudio como el modo de expresar nuestro conocimiento incompleto acerca de tal sistema: las probabilidades son concebidas, entonces, como una medida de nuestra ignorancia acerca el microestado preciso en el que se encuentra el sistema y, por lo tanto, su introducción es resultado de la inevitable imprecisión de nuestras mediciones. Es justamente esta interpretación de la probabilidad lo que ha originado las acusaciones de subjetividad que tradicionalmente se han dirigido al enfoque gibbsiano.

Una vez definido el ensemble representativo del sistema bajo estudio, el enfoque de Gibbs brinda una descripción dinámica de su evolución: en la medida en que cada uno de los sistemas del ensemble es un sistema mecánico, evolucionará a través del tiempo de acuerdo con las leyes dinámicas de la mecánica clásica. El enfoque de Boltzmann, en cambio, al adoptar como punto de partida una aproximación combinatoria, en principio no suministra una explicación dinámica del comportamiento del sistema. Cuando se intenta dar cuenta, en términos de la dinámica subyacente, de la evolución al equilibrio en el marco boltzmanniano, aparecen las dificultades que, ya desde los orígenes de la teoría, dieron lugar a los debates en torno al problema del llamado "desorden molecular" (cfr. Culverwell 1894, Burbury 1894, Boltzmann 1895a, 1895 b). Actualmente, los defensores del enfoque de Boltzmann pretenden prescindir de la dinámica subyacente apelando al concepto de comportamiento "típico" del sistema: 
$S_{B}$ típicamente aumentará de un modo que explica y describe cualitativamente la evolución hacia el equilibrio de los sistemas macroscópicos. Típico, como es usado aquí, significa que el conjunto de microestados correspondientes a un macroestado $M$ dado, para el cual la evolución conduce a una disminución macroscópica de la entropía de Boltzmann durante cierto periodo fijo $\tau$, ocupa un subconjunto de $\Gamma_{M}$ cuyo volumen de Liouville es una fracción de $\left|\Gamma_{M}\right|$ [de la medida $\mu\left(\Gamma_{M}\right)$ en nuestra terminología] que tiende muy rápidamente (exponencialmente) a cero cuando aumenta el número de átomos en el sistema. (Lebowitz 1999, p. S348)

Este sentido de "típico" se define en términos de la relación entre las medidas de diferentes regiones del espacio de las fases, independientemente de toda consideración dinámica. Sin embargo, tal como algunos autores señalan ( $c f r$. Lavis 2005), este modo de caracterizar la noción de "típico" no resulta suficiente para calificar una trayectoria en el espacio de las fases como "típica": ello exige, una vez más, introducir consideraciones dinámicas, originalmente ausentes en el marco boltzmanniano. ${ }^{16}$

Por último, los enfoques de Boltzmann y de Gibbs difieren en cuanto al nivel descriptivo adoptado como punto de partida teórico. El enfoque de Boltzmann puede caracterizarse como un enfoque macro-micro puesto que, desde el comienzo, requiere la introducción de alguna -o algunas - variable(s) macroscópica(s) en función de la(s) cual(es) se definirán los macroestados y sus correspondientes regiones representativas en el espacio de las fases. Por el contrario, el enfoque de Gibbs adopta la estrategia inversa, micro-macro: se parte de una caracterización microscópica de los elementos del ensemble y, en un paso lógicamente posterior, el valor de las macrovariables se asocia con el promedio en fase de magnitudes definidas sobre el ensemble. Dado que, desde esta perspectiva, las variables macroscópicas no se han presupuesto, la coincidencia entre sus valores y los promedios en fase no es un resultado que haya de cumplirse necesariamente; en efecto, en ciertas condiciones es posible que los métodos de Gibbs "no funcionen", en particular, si el sistema no es ergódico (para una discusión sobre este punto, cfr. Lombardi 2003).

${ }^{16}$ Lavis (2005, pp. 258-260) pone de manifiesto que, cuando se define con precisión la noción de "típico" aplicada a trayectorias, la ergodicidad aparece como una condición suficiente para que las trayectorias típicas se comporten de un modo termodinámico. Esta conclusión constituye un interesante argumento contra los defensores del enfoque de Boltzmann, quienes insisten en que la ergodicidad no es condición necesaria ni suficiente para la irreversibilidad. 
Estas diferencias en los conceptos que se encuentran a la base de las perspectivas boltzmanniana y gibbsiana hacen muy difícil subsumir ambos enfoque bajo un único marco teórico. Lavis (2005), por ejemplo, intenta la reconciliación mediante una redefinición del concepto de equilibrio: las propiedades dicotómicas del sistema de "estar en equilibrio" o "no estar en equilibrio" se reemplazan por una propiedad continua de "ser común" [commonness]: un microestado es más o menos común según sea mayor o menor la medida del macroestado al cual pertenece. No obstante, esta estrategia no parece dar cuenta de la idea termodinámica de un sistema que ha alcanzado una situación donde sus variables macroscópicas ya no experimentan variaciones con el tiempo. Nuestra sospecha es que si la reconciliación entre los enfoques de Boltzmann y de Gibbs es posible, ésta deberá basarse en una aproximación "algebraica" de la mecánica estadística, a la manera del enfoque algebraico de la mecánica cuántica: el álgebra de observables se adopta como punto de partida, y las magnitudes básicas pasan a ser los valores medios de tales observables. Desde esta perspectiva, la dinámica relevante es la correspondiente a tales valores medios y no la del estado del sistema; pero la exploración de esta posibilidad excede completamente los límites del presente trabajo.

En definitiva, el hecho de que los enfoques de Boltzmann y de Gibbs brinden resultados prácticamente coincidentes en el caso de los sistemas tradicionalmente estudiados en mecánica estadística no debe interpretarse como si ambos pudieran identificarse bajo ciertas condiciones definidas. Las discrepancias entre las bases conceptuales de las propuestas boltzmanniana y gibbsiana son tan profundas que la búsqueda de una reconciliación teórica entre ambos enfoques continúa presentándose como un problema abierto en los fundamentos de la mecánica estadística.

\section{Conclusiones}

Si bien la mecánica estadística es una de las teorías más tradicionales de la física, aún hoy sigue generando debates en torno a sus fundamentos. El origen de los desacuerdos reside en la coexistencia de dos enfoques teóricos, el de Boltzmann y el de Gibbs, que difieren en aspectos conceptuales centrales, en particular, en las condiciones y el modo de dar cuenta de la irreversibilidad. En la actualidad, los defensores de ambos enfoques se enfrentan desacreditando el enfoque contrario mediante argumentos teóricos y contraejemplos particulares. Sin embargo, los participantes del debate suelen pasar por alto un hecho central: las perspectivas boltzmanniana y gibbsiana no brindan 
explicaciones alternativas de un único concepto de irreversibilidad, sino que cada uno de los enfoques define su propio concepto de equilibrio y, con ello, de irreversibilidad.

En el presente trabajo se ha intentado presentar con claridad las divergencias conceptuales entre los enfoques de Boltzmann y de Gibbs, a menudo ocultas en presentaciones que yuxtaponen elementos provenientes de ambas perspectivas en un intento por brindar una visión orgánica y unificada de la mecánica estadística. Esta tarea ha permitido disolver el debate en su forma actual, al poner de manifiesto los diferentes conceptos de irreversibilidad utilizados en el desarrollo de la discusión. Por último se ha argumentado que, en los casos de interés práctico, tanto el enfoque de Boltzmann como el de Gibbs brindan resultados adecuados, aun cuando ello no cancele las diferencias básicas entre ambas perspectivas teóricas.

\section{BIBLIOGRAFÍA}

Arnold, V.I. y A. Avez, 1968, Ergodic Problems of Statistical Mechanics, Benjamin, Nueva York.

Blaise, P., P. Durand y O.H. Rousseau, 1994, "Irreversible Evolution towards Equilibrium of Coupled Quantum Harmonic Oscillators. A Coarse Grained Approach", Physica A, vol. 209, pp. 51-82.

Boltzmann, L., 1895a, "On Certain Questions of the Theory of Gases", Nature, vol. 51, pp. 413-415.

- 1895b, "On the Minimum Theorem in the Theory of Gases", Nature, vol. 52, p. 221.

Bricmont, J., 1995, "Science of Chaos or Chaos in Science?", Physicalia Magazine, vol. 17, pp. 159-208.

Brush, S., 1976, The Kind of Motion We Call Heat, North Holland, Amsterdam.

Burbury, S.H., 1894, "The Kinetic Theory of Gases", Nature, vol. 51, pp. 175-176.

Callender, C., 1999, "Reducing Thermodynamics to Statistical Mechanics: The Case of Entropy", Journal of Philosophy, vol. 96, pp. 348-373.

Culverwell, E.P., 1894, “Dr. Watson's Proof of Boltzmann's Theorem on Permanence of Distributions", Nature, vol. 50, p. 617.

Driebe, D.J., 1994, "Letters" (respuesta a Lebowitz 1993), Physics Today, vol. 47, pp. 14-15.

Earman, J. y M. Rédei, 1996, "Why Ergodic Theory Does Not Explain the Success of Equilibrium Statistical Mechanics", British Journal for the Philosophy of Science, vol. 47, pp. 63-78.

Friedman, K.S., 1976, “A Partial Vindication of Ergodic Theory", Philosophy of Science, vol. 43, pp. 151-162. 
Gaioli, F.H. y E.T. García-Alvarez, 1999, "Exact Time Evolution in Harmonic Quantum Brownian Motion", Physica A, vol. 264, pp. 338-344.

Gibbs, J.W., 1902, Elementary Principles in Statistical Mechanics, Yale University Press, New Haven.

Guttmann, Y.M., 1999, The Concept of Probability in Statistical Physics, Cambridge University Press, Cambridge.

Hollinger, H.B. y M.J. Zenzen, 1982, “An Interpretation of Macroscopic Irreversibility within the Newtonian Framework", Philosophy of Science, vol. 49, pp. 309-354.

Kac, M., 1959, Probability and Related Topics in the Physical Sciences, Interscience Publishers, Nueva York.

Krylov, N.S., 1979, Works on the Foundations of Statistical Physics, Princeton University Press, Princeton.

Lavis, D.A., 2005, "Boltzmann and Gibbs; An Attempted Reconciliation", Studies in History and Philosophy of Modern Physics, vol. 36, pp. 245273.

Lebowitz, J.L., 1999, "Statistical Mechanics: A Selective Review of Two Central Issues", Reviews of Modern Physics, vol. 71, pp. S346-S357.

—_, 1994, "Lebowitz Replies", Physics Today, vol. 47, pp. 115-116.

, 1993, “Boltzmann's Entropy and Time’s Arrow", Physics Today, vol. 46, pp. 32-38.

Lebowitz, J.L. y O. Penrose, 1973, "Modern Ergodic Theory", Physics Today, vol. 26, pp. 23-29.

Leeds, S., 1989, "Malament and Zabell on Gibbs Phase Averages", Philosophy of Science, vol. 56, pp. 325-340.

Lombardi, O., 2003, "El problema de la ergodicidad en mecánica estadística", Crítica. Revista Hispanoamericana de Filosofía, vol. 35, pp. 3-41.

Malament, D.B. y S.L. Zabell, 1980, "Why Gibbs Phase Averages Work -The Role of Ergodic Theory", Philosophy of Science, vol. 47, pp. 339349.

Nicolis, G. e I. Prigogine, 1989, Exploring Complexity. An Introduction, W.H. Freeman, Nueva York.

Prigogine, I. e I. Stengers, 1991, Entre el tiempo y la eternidad, Alianza Editorial, Buenos Aires.

_- 1990, La nueva alianza. Metamorfosis de la ciencia, Alianza Editorial, Madrid.

Tolman, R.C., 1938, The Principles of Statistical Mechanics, Clarendon Press, Oxford.

Recibido el 15 de febrero de 2005; revisado el 20 de septiembre de 2005; aceptado el 7 de diciembre de 2005. 